\title{
Fundamental groups of line arrangements: Enumerative aspects
}

\begin{abstract}
Alexander I. Suciu
ABSTRACT. This is a survey of some recent developments in the study of complements of line arrangements in the complex plane. We investigate the fundamental groups and finite covers of those complements, focusing on homological and enumerative aspects.

The unifying framework for this study is the stratification of the character variety of the fundamental group, $G$, by the jumping loci for cohomology with coefficients in rank 1 local systems. Counting certain torsion points on these "characteristic" varieties yields information about the homology of branched and unbranched covers of the complement, as well as on the number of lowindex subgroups of its fundamental group.

We conclude with two conjectures, expressing the lower central series quotients of $G / G^{\prime \prime}$ (and, in some cases, $G$ itself) in terms of the closely related "resonance" varieties. We illustrate the discussion with a number of detailed examples, some of which reveal new phenomena.
\end{abstract}

\section{Contents}

1. Introduction 2

2. Fundamental groups and Alexander matrices 5

3. Resonance varieties and characteristic varieties 9

\begin{tabular}{lll}
\hline 4. Homology of finite-index subgroups and torsion points on varieties & 12
\end{tabular}

$\begin{array}{llll}5 . \quad \text { Congruence covers and polynomial periodicity } & 13\end{array}$

\begin{tabular}{ll|l}
\hline 6. & Hirzebruch covering surfaces & 15
\end{tabular}

$\begin{array}{ll}7 . \quad \text { Counting finite-index subgroups } & 17\end{array}$

$\begin{array}{lll}\text { 8. Lower central series quotients and Chen groups } & 19\end{array}$

9. Guide to examples 23

\begin{tabular}{ll|l}
$10 . \quad$ Examples & 25
\end{tabular}

References 34

2000 Mathematics Subject Classification. Primary 14F35, 32S22, 52C35, 57M05; Secondary 20E07, 20F14, 20J05.

Key words and phrases. hyperplane arrangement, fundamental group, Alexander matrix, characteristic variety, resonance variety, congruence cover, Hirzebruch covering surface, polynomial periodicity, finite-index subgroup, Hall invariant, lower central series, Chen groups.

(C)0000 (copyright holder) 


\section{Introduction}

In the introduction to [37], Hirzebruch wrote: "The topology of the complement of an arrangement of lines in the projective plane is very interesting, the investigation of the fundamental group of the complement very difficult." Much progress has occurred since that assessment was made in 1983. The fundamental groups of complements of line arrangements are still difficult to study, but enough light has been shed on their structure, that once seemingly intractable problems can now be attacked in earnest. This paper is meant as an introduction to some recent developments, and as an invitation for further investigation. We take a fresh look at several topics studied in the past two decades, from the point of view of a unified framework. Though most of the material is expository, we provide new examples and applications, which in turn raise several questions and conjectures.

1.1. Hyperplane arrangements. In its simplest manifestation, an arrangement is merely a finite collection of lines in the real plane. These lines cut the plane into pieces, and understanding the topology of the complement amounts to counting those pieces. In the case of lines in the complex plane (or, for that matter, hyperplanes in complex $\ell$-space), the complement is connected, and its topology (as reflected, for example, in its fundamental group) is much more interesting.

An important example is the braid arrangement of diagonal hyperplanes in $\mathbb{C}^{\ell}$. In that case, loops in the complement can be viewed as (pure) braids on $\ell$ strings, and the fundamental group can be identified with the pure braid group $P_{\ell}$. For an arbitrary hyperplane arrangement, $\mathcal{A}=\left\{H_{1}, \ldots, H_{n}\right\}$, with complement $X(\mathcal{A})=$ $\mathbb{C}^{\ell} \backslash \bigcup_{i=1}^{n} H_{i}$, the identification of the fundamental group, $G(\mathcal{A})=\pi_{1}(X(\mathcal{A}))$, is more complicated, but it can be done algorithmically, using the theory of braids (see [59, 7], and the references therein). This theory, in turn, is intimately connected with the theory of knots and links in 3-space, with its wealth of algebraic and combinatorial invariants, and its varied applications to biology, chemistry, and physics. A revealing example where developments in arrangement theory have influenced knot theory is Falk and Randell's 19 proof of the residual nilpotency of the pure braid group, a fact that has been put to good use in the study of Vassiliev invariants. We refer to the excellent surveys 18, 20 for a more complete treatment of the homotopy theory of arrangements.

A more direct link to physics is provided by the deep connections between arrangement theory and hypergeometric functions. Work by Schechtman-Varchenko 68 and many others has profound implications in the study of Knizhnik-Zamolodchikov equations in conformal field theory. We refer to the recent monograph by Orlik and Terao [60] for a comprehensive account of this fascinating subject.

Hyperplane arrangements, and the closely related configuration spaces, are used in numerous areas, including robotics, graphics, molecular biology, computer vision, and databases for representing the space of all possible states of a system characterized by many degrees of freedom. Understanding the topology of complements of subspace arrangements and configuration spaces is important in robot motion planning (finding a collision-free motion between two placements of a given robot among a set of obstacles, see [25]), and in multi-dimensional billiards (describing periodic trajectories of a mass-point in a domain in Euclidean space with reflecting boundary, see 21]). 
1.2. Characteristic and resonance varieties. The unifying framework for the study of fundamental groups of hyperplane arrangements is provided by their characteristic and resonance varieties. The origins of those varieties can be traced back to the work of Novikov [58] in the mid 1980's on the cohomology of smooth manifolds with coefficients in local systems, and its relation to Morse theory for 1forms. Novikov showed that the Betti numbers remain constant almost everywhere on the representation space of the fundamental group of the manifold, but they increase on the union of countably many algebraic submanifolds.

The simplest situation is when the fundamental group of the manifold, $G=$ $\pi_{1}(X)$, has abelianization $H_{1}(G) \cong \mathbb{Z}^{n}$, and the rank of the local coefficient system is 1 . Then, the representation variety is the algebraic torus $\operatorname{Hom}\left(G, \mathbb{C}^{*}\right) \cong \mathbb{C}^{* n}$, and the jumping loci for 1-dimensional cohomology are the characteristic varieties of $G$,

$$
V_{d}(G, \mathbb{C})=\left\{\mathbf{t} \in \mathbb{C}^{* n} \mid \operatorname{dim}_{\mathbb{C}} H^{1}\left(G, \mathbb{C}_{\mathbf{t}}\right) \geq d\right\}, \quad 1 \leq d \leq n .
$$

Work of Libgober [43], Hironaka [34, 35], and Sakuma [66] clarified the connection between the characteristic varieties, the Alexander matrix, and the Betti numbers of finite abelian covers. []

A breakthrough occurred in 1997, when Arapura [1] showed that the characteristic varieties of $G=\pi_{1}(X)$ are unions of (possibly torsion-translated) subtori of $\mathbb{C}^{* n}$, provided $X$ is the complement of a normal-crossing divisor in a compact Kähler manifold with vanishing first Betti number.P In particular, this result applies to the characteristic varieties of an arrangement group, $G=G(\mathcal{A})$. Also in 1997, Falk 15 defined the resonance varieties of the Orlik-Solomon algebra $H^{*}(X(\mathcal{A}), \mathbb{C})$. There ensued a flurry of activity, showing (in $[10,44,4,45]$ ) that these varieties are the tangent cones at the origin to the characteristic varieties. The combinatorial description of the resonance varieties of arrangements, started by Falk, was completed by Libgober and Yuzvinsky 46 .

1.3. Betti numbers of finite covers. One of the reasons for studying the characteristic varieties of the group $G=\pi_{1}(X)$ is the precise information they give about the (rational) homology of covering spaces of $X$. Indeed, suppose $X^{\prime} \rightarrow X$ is a regular cover, with finite, abelian Galois group. Results of Libgober 43, Sakuma [66], and Hironaka [36] show how to compute $b_{1}\left(X^{\prime}\right)$ by counting torsion points of a certain order on the algebraic torus $\operatorname{Hom}\left(G, \mathbb{C}^{*}\right)$, according to their depth in the filtration by the varieties $V_{d}(G, \mathbb{C})$. (Explicit formulas of this sort are given in Corollary 4.5 and Theorem 5.2.)

Of particular interest is the case of congruence covers, $X_{N}$, defined by the canonical projection $G \rightarrow H_{1}\left(G, \mathbb{Z}_{N}\right)$. As noted by Sarnak and Adams [67, the sequence of Betti numbers $\left\{b_{1}\left(X_{N}\right)\right\}_{N \in \mathbb{N}}$ is polynomially periodic, i.e., there exists polynomials $P_{1}(x), \ldots, P_{T}(x)$, such that $b_{1}\left(X_{N}\right)=P_{i}(N)$, whenever $N \equiv i \bmod T$. (This follows from Sakuma's formula, by means of a deep result of Laurent on torsion points on varieties). Here we give examples of arrangements $\mathcal{A}$ for which the

\footnotetext{
${ }^{1}$ In fact, one can argue that the origins of characteristic varieties (so named in 44 ) go back to the work of James Alexander, in the early 1920's. Indeed, for a knot in $\mathbb{S}^{3}$, the characteristic varieties are nothing but the roots of the Alexander polynomial; if the knot is fibered, those are the eigenvalues of the algebraic monodromy, whence the name for those varieties (also known as Alexander varieties 35 )

${ }^{2}$ Arapura's theorem works for higher-rank representations of $\pi_{1}(X)$, as well as for the jumping loci of higher-dimensional cohomology groups of $X$.
} 
minimal period, $T$, is greater than 1 (in fact, $T=2$ ). This non-trivial periodicity in the Betti numbers of congruence covers of $X(\mathcal{A})$ can be traced back to the presence of isolated torsion points in $V_{2}(G(\mathcal{A}), \mathbb{C})$.

Similar techniques apply to the study of Hirzebruch covering surfaces ${ }^{3}, M_{N}(\mathcal{A})$, defined in [37, as follows. Let $\mathcal{A}$ be a central arrangement of planes in $\mathbb{C}^{3}$, and let $\overline{\mathcal{A}}$ be the corresponding arrangement of lines in $\mathbb{C P}^{2}$. Then $M_{N}(\mathcal{A})$ is the minimal desingularization of the branched congruence cover, $\widehat{X}_{N}(\overline{\mathcal{A}})$, of the projective plane, ramified along $\overline{\mathcal{A}}$. The polynomial periodicity of the sequence $\left\{b_{1}\left(M_{N}(\mathcal{A})\right\}_{N \in \mathbb{N}}\right.$ was established by Hironaka [33] and Sakuma [66]. We give examples of arrangements for which the minimal period is $T=4$. The non-trivial periodicity in the Betti numbers of Hirzebruch covering surfaces is due to the presence of torsion-translated, positive-dimensional components in $V_{1}(G(\mathcal{A}), \mathbb{C})$.

1.4. Characteristic varieties over finite fields. The usefulness of characteristic varieties in the study of finite abelian covers $X^{\prime} \rightarrow X$ is limited by the fact that they only give the rank of $H_{1}\left(X^{\prime}\right)$, but not its torsion coefficients. This limitation can be overcome (at least partially) by considering the characteristic varieties of $G=\pi_{1}(X)$ over arbitrary fields $\mathbb{K}$. These varieties were first considered by Matei in his thesis [52]; the analogous resonance varieties over finite fields were first studied in [54. From the stratification of $\operatorname{Hom}(G, \mathbb{K})$ by the varieties $V_{d}(G, \mathbb{K})$, one can derive information about the torsion in $H_{1}\left(X^{\prime}\right)$, away from the prime $q=$ char $\mathbb{K}$. Such a method (which recovers and extends results of Libgober, Sakuma, and Hironaka) was recently developed in [55. We sketch this method here, and mention some of its applications, which show why it is important to look beyond Betti numbers.

In [23], Fox showed how to compute the number of metacyclic representations of link groups, by means of his free differential calculus. In [55], we generalize Fox's method, and interpret it in terms of characteristic varieties over certain Galois fields $\mathbb{K}$. This provides a way to count homomorphisms from a finitely-presented group $G$ onto certain finite metabelian groups, such as the symmetric group $S_{3}$ and the alternating group $A_{4}$. In turn, this count gives information about the number of finite-index subgroups of $G$, at least for low index. All these enumeration problems reduce to counting torsion points on the characteristic varieties $V_{d}(G, \mathbb{K})$. Such a count depends in subtle ways on the field $\mathbb{K}$, and is certainly affected by the presence of translated subtori in $V_{d}(G, \mathbb{C})$.

1.5. Lower central series. Probably the most studied numerical invariants of an arrangement group $G$ are the ranks of its lower central series quotients, $\phi_{k}(G)=\operatorname{rank} \gamma_{k} G / \gamma_{k+1} G$. The impetus came from the work of Kohno [40], who used rational homotopy theory to compute $\phi_{k}\left(P_{\ell}\right)$, the LCS ranks of the braid arrangement group. Falk and Randell [17], using more direct methods, established the celebrated $L C S$ formula for the broader class of fiber-type arrangements, expressing $\phi_{k}(G(\mathcal{A}))$ in terms of the exponents of the arrangement $\mathcal{A}$. Recent work by a number of authors, [69, 8, 39, 61, 62], has further clarified the meaning of this formula, and greatly expanded its range of applicability.

Another direction was started in [6, 9], with the study of the ranks of Chen groups of arrangements, $\theta_{k}(G)=\phi_{k}\left(G / G^{\prime \prime}\right)$. The $\theta$-invariants can provide stronger

\footnotetext{
${ }^{3}$ Not to be confused with the Hirzebruch surfaces, defined 30 years previously.
} 
information than the $\phi$-invariants, distinguishing, in some cases, groups of fibertype arrangements from the corresponding direct products of free groups.

A number of experiments (some of which we record here) has revealed tantalizing parallels to other, seemingly unrelated objects associated to the group $G$-namely, its resonance varieties. As a result, we formulate two conjectures, expressing the ranks of the LCS quotients of $G / G^{\prime \prime}$ (and, in some cases, $G$ itself) in terms of the dimensions of the components of the resonance variety $R_{1}(G, \mathbb{C})$.

1.6. Organization of the paper. Sections 2 and 3 contain basic material on complements of complex hyperplane arrangements, their fundamental groups, Alexander matrices, and resonance and characteristic varieties. Much of the exposition is based on joint work with D. Cohen [7, 9, 10] and D. Matei [53, 54, 55].

In Section 4, we discuss the connection between the characteristic varieties of a group and the homology of its finite-index subgroups. We use this connection in Section 7 to count low-index subgroups. These two sections are a partial summary of a recent paper with D. Matei [55].

In Sections 5 and 6 , we study congruence covers and Hirzebruch covering surfaces, and the polynomial periodicity of their Betti numbers.

In Section 8, we discuss the LCS quotients and Chen groups of an arrangement group, and their possible connection to the resonance varieties.

Section 10 contains about a dozen examples worked out in detail. A guide to the examples is provided in Section 9 .

Acknowledgments. I wish to thank Dan Cohen, Mike Falk, Anatoly Libgober, Daniel Matei, Ştefan Papadima, Dick Randell, and Sergey Yuzvinsky for useful discussions and comments while some of this material was being developed. Especially, Dan Cohen, who attracted me to the subject, and Daniel Matei, together with whom a good deal of the work presented here was done.

I also wish to thank Emma Previato, whose encouragement and enthusiasm convinced me to undertake this project, and the referee, for pertinent remarks.

A preliminary version of the paper was presented in the Special Session on Enumerative Geometry in Physics, at the AMS Spring Eastern Section Meeting, held in Lowell, MA in April 2000. A fuller version was presented as an Invited Address at the AMS Fall Eastern Section Meeting, held in New York, NY in November 2000.

\section{Fundamental groups and Alexander matrices}

2.1. Arrangements of hyperplanes. A (complex) hyperplane arrangement is a finite set, $\mathcal{A}$, of codimension- 1 affine subspaces in a finite-dimensional complex vector space, $\mathbb{C}^{\ell}$. The main combinatorial object associated to $\mathcal{A}$ is its intersection lattice, $\mathcal{L}(\mathcal{A})=\left\{\emptyset \neq \bigcap_{H \in \mathcal{B}} H \mid \mathcal{B} \subseteq \mathcal{A}\right\}$. This is a ranked poset, consisting of all non-empty intersections of $\mathcal{A}$, ordered by reverse inclusion, and with rank function given by codimension. The main topological object associated to $\mathcal{A}$ is its complement, $X(\mathcal{A})=\mathbb{C}^{\ell} \backslash \bigcup_{H \in \mathcal{A}} H$. This is an open, $\ell$-dimensional complex manifold, whose topological invariants are intimately connected to the combinatorics of the arrangement. A basic reference for the subject is the book by Orlik and Terao 59.

EXAMPLE 2.2. Probably the best-known hyperplane arrangement is the braid arrangement in $\mathbb{C}^{\ell}$. This is the complexification of the $\mathrm{A}_{\ell-1}$-reflection arrangement, $\mathcal{B}_{\ell}=\left\{\operatorname{ker}\left(z_{i}-z_{j}\right)\right\}_{1 \leq i<j \leq \ell}$, with lattice $\mathcal{L}\left(\mathcal{B}_{\ell}\right)=\Pi(\ell)$, the partition lattice of 
$[\ell]:=\{1, \ldots, \ell\}$, complement $X\left(\mathcal{B}_{\ell}\right)=F(\ell, \mathbb{C})$, the configuration space of $\ell$ ordered points in $\mathbb{C}$, and group $\pi_{1}\left(X\left(\mathcal{B}_{\ell}\right)\right)=P_{\ell}$, the pure braid group on $\ell$ strings.

Let $\mathcal{A}=\left\{H_{1}, \ldots, H_{n}\right\}$ be a central arrangement in $\mathbb{C}^{\ell}$ (that is, $\mathbf{0} \in \bigcap_{i} H_{i}$ ). A defining polynomial for $\mathcal{A}$ may be written as $Q_{\mathcal{A}}=f_{1} \cdots f_{n}$, where $f_{i}$ are distinct linear forms, with $H_{i}=\operatorname{ker} f_{i}$. Choose coordinates $\left(z_{1}, \ldots, z_{\ell}\right)$ in $\mathbb{C}^{\ell}$ so that $H_{n}=\left\{z_{\ell}=0\right\}$. The corresponding decone of $\mathcal{A}$ is the affine arrangement $\mathcal{A}^{*}=\mathbf{d} \mathcal{A}$ in $\mathbb{C}^{\ell-1}$, with defining polynomial $Q_{\mathcal{A}^{*}}=Q_{\mathcal{A}}\left(z_{1}, \ldots, z_{\ell-1}, 1\right)$. Reversing the procedure yields $\mathcal{A}=\mathbf{c} \mathcal{A}^{*}$, the cone of $\mathcal{A}^{*}$. The respective complements are related as follows: $X(\mathcal{A}) \cong X\left(\mathcal{A}^{*}\right) \times \mathbb{C}^{*}$, where $\mathbb{C}^{*}=\mathbb{C} \backslash\{0\}$.

2.3. Arrangements of lines. We will be interested here in the low-dimensional topology of arrangements - mainly in questions related to the fundamental group of the complement, and the cohomology ring up to degree 2. For that, we may restrict our attention to (affine) line arrangements in $\mathbb{C}^{2}$, or, by coning, to (central) plane arrangements in $\mathbb{C}^{3}$. Indeed, if $\mathcal{A}$ is a hyperplane arrangement, with complement $X$, let $\mathcal{A}^{\prime}$ be a generic two-dimensional section of $\mathcal{A}$, with complement $X^{\prime}$. Then, by the Lefschetz-type theorem of Hamm and Lê [31], the inclusion $j: X^{\prime} \rightarrow X$ induces an isomorphism $j_{*}: \pi_{1}\left(X^{\prime}\right) \rightarrow \pi_{1}(X)$ and a monomorphism $j^{*}: H^{2}(X) \rightarrow H^{2}\left(X^{\prime}\right)$. By the Brieskorn-Orlik-Solomon theorem (cf. [59]), the map $j^{*}$ is, in fact, an isomorphism, and thus $H^{\leq 2}(X) \cong H^{\leq 2}\left(X^{\prime}\right)$. Furthermore, the Hurewicz homomorphism $\pi_{2}(X) \rightarrow H_{2}(X)$ is the zero map, see Randell 64. Thus, by a theorem of Hopf, $H^{\leq 2}\left(\pi_{1}(X)\right) \cong H^{\leq 2}(X)$, see [54.

So let $\mathcal{A}=\left\{l_{1}, \ldots, l_{n}\right\}$ be an arrangement of $n$ affine lines in $\mathbb{C}^{2}$. Let $v_{1}, \ldots, v_{s}$ be the intersection points of the lines. If $v_{q}=l_{i_{1}} \cap \cdots \cap l_{i_{r}}$, set $I_{q}=\left\{i_{1}, \ldots, i_{r}\right\}$. Let $\mathcal{L}_{1}(\mathcal{A})=[n]$ and $\mathcal{L}_{2}(\mathcal{A})=\left\{I_{1}, \ldots, I_{s}\right\}$. The combinatorics of the arrangement is encoded in its intersection poset, $\mathcal{L}(\mathcal{A})=\left\{\mathcal{L}_{1}(\mathcal{A}), \mathcal{L}_{2}(\mathcal{A})\right\}$, which keeps track of the incidence relations between the points and the lines of the arrangement.

From $\mathcal{L}(\mathcal{A})$, one can extract some simple, but very useful numerical information. First note that $n=\left|\mathcal{L}_{1}(\mathcal{A})\right|, s=\left|\mathcal{L}_{2}(\mathcal{A})\right|$. Let

$$
m_{r}=\#\left\{I \in \mathcal{L}_{2}(\mathcal{A})|| I \mid=r\right\}
$$

be the number of intersection points of multiplicity $r$. Note that $\sum_{r} m_{r}=s$. The homology groups of the complement, $X=X(\mathcal{A})$, are easily computable in terms of these data (see [59]):

$$
H_{1}(X)=\mathbb{Z}^{n}, \quad H_{2}(X)=\mathbb{Z}^{\sum_{r} m_{r}(r-1)}, \quad H_{i}(X)=0, \text { for } i>2 .
$$

2.4. Fundamental groups. We now describe a procedure for finding a finite presentation for the fundamental group of the complement of an arrangement, $G(\mathcal{A})=\pi_{1}(X(\mathcal{A}))$. The information encoded in the intersection lattice is not a priori enough; we need slightly more information, encoded in the braid monodromy of the arrangement. As shown in [7], the resulting presentation is Tietze-I equivalent to the presentations of Randell [63] and Arvola [1].

Let $B_{n}$ be the Artin braid group on $n$ strings, with generators $\sigma_{1}, \ldots, \sigma_{n-1}$. Let $P_{n}=\operatorname{ker}\left(B_{n} \rightarrow S_{n}\right)$ be the subgroup of braids with trivial permutation of the strings, with generators $A_{i j}=\sigma_{j-1} \cdots \sigma_{i+1} \sigma_{i}^{2} \sigma_{i+1}^{-1} \cdots \sigma_{j-1}^{-1}(1 \leq i<j \leq n)$. The pure braid group acts on the free group $F_{n}=\left\langle x_{1}, \ldots, x_{n}\right\rangle$ by restricting the Artin 
representation $B_{n} \hookrightarrow \operatorname{Aut}\left(F_{n}\right)$ to $P_{n}$. Explicitly:

$$
A_{i j}\left(x_{r}\right)= \begin{cases}x_{r} & \text { if } r<i \text { or } r>j, \\ x_{i} x_{r} x_{i}^{-1} & \text { if } r=j, \\ x_{i} x_{j} x_{r} x_{j}^{-1} x_{i}^{-1} & \text { if } r=i, \\ {\left[x_{i}, x_{j}\right] x_{r}\left[x_{i}, x_{j}\right]^{-1}} & \text { if } i<r<j,\end{cases}
$$

see Birman [3]. For an increasingly ordered set $I=\left\{i_{1}, \ldots, i_{r}\right\} \subset[n]$, let $\breve{I}=$ $\left\{i_{1}, \ldots, i_{r-1}\right\}$, and let

$$
A_{I}=A_{i_{1} i_{2}} A_{i_{1} i_{3}} A_{i_{2} i_{3}} A_{i_{1} i_{4}} A_{i_{2} i_{4}} A_{i_{3} i_{4}} \cdots A_{i_{1} i_{r}} \cdots A_{i_{r-1} i_{r}}
$$

be the braid in $P_{n}$ which performs a full twist on the strands corresponding to $I$, leaving the other strands fixed.

Now choose a generic linear projection $p: \mathbb{C}^{2} \rightarrow \mathbb{C}$, and a point $y_{0} \in \mathbb{C}$, with $\operatorname{Re}\left(y_{0}\right) \gg 0$. Let $l_{0}$ be the line $p^{-1}\left(y_{0}\right)$. Label the lines $l_{i}$ and the vertices $v_{q}$ so that $\operatorname{Re}\left(p\left(v_{1}\right)\right)>\cdots>\operatorname{Re}\left(p\left(v_{s}\right)\right)$ and $\operatorname{Re}\left(l_{0} \cap l_{1}\right)<\cdots<\operatorname{Re}\left(l_{0} \cap l_{n}\right)$. Pick a basepoint $\tilde{y}_{0} \in \mathbb{C}^{2}$ so that $\tilde{y}_{0} \in l_{0}$, but $\tilde{y}_{0} \notin l_{i}$. Let $x_{i}$ be the meridian loops to $l_{i}$, based at $\tilde{y}_{0}$, and oriented compatibly with the complex orientations of $l_{i}$ and $\mathbb{C}^{2}$. There are then pure braids

$$
\alpha_{1}=A_{I_{1}}^{\delta_{1}}, \ldots, \alpha_{s}=A_{I_{s}}^{\delta_{s}},
$$

where $a^{b}:=b^{-1} a b$, such that $G=G(\mathcal{A})$ has braid monodromy presentation

$$
\left.G=G\left(\alpha_{1}, \ldots, \alpha_{s}\right):=\left\langle x_{1}, \ldots, x_{n}\right| \alpha_{q}\left(x_{i}\right)=x_{i} \text { for } i \in \check{I}_{q} \text { and } q \in[s]\right\rangle .
$$

The conjugating braids $\delta_{q}$ may be obtained as follows. In the case where $\mathcal{A}$ is the complexification of a real arrangement, each vertex set $I_{q}$ gives rise to a partition $[n]=I_{q}^{\prime} \cup I_{q} \cup I_{q}^{\prime \prime}$ into lower, middle, and upper indices. Let $J_{q}=\left\{i \in I_{q}^{\prime \prime} \mid \min I_{q}<\right.$ $\left.i<\max I_{q}\right\}$. Then $\delta_{q}$ is the subword of the full twist $A_{12 \ldots n}=\prod_{i=2}^{n} \prod_{j=1}^{i-1} A_{j i}$, given by

$$
\delta_{q}=\prod_{i \in I_{q}} \prod_{j \in J_{q}} A_{j i},
$$

see 34, 11, 7]. In the general case, the braids $\delta_{q}$ can be read off a "braided wiring diagram" associated to $\mathcal{A}$ and the projection $p$, see $[7]$ for further details.
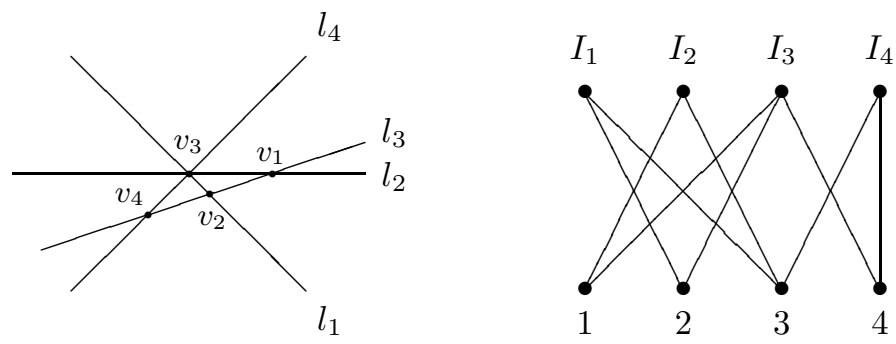

FiguRE 1. An arrangement of 4 lines in $\mathbb{C}^{2}$ and its intersection poset

EXAMPLE 2.5. Here is a simple example which illustrates the procedure. Let $\mathcal{A}$ be the arrangement of 4 lines in $\mathbb{C}^{2}$ defined by $Q_{\mathcal{A}}=z(z-y)(z+y)(2 z-y+1)$. The lines $l_{1}, l_{2}, l_{3}, l_{4}$, together with the vertices $v_{1}, v_{2}, v_{3}, v_{4}$ are depicted in Figure 1 . 
The intersection lattice is also shown. Take $p: \mathbb{C}^{2} \rightarrow \mathbb{C}$ to be the projection $p(y, z)=y$, and choose $y_{0}=2$. The corresponding braid monodromy generators are: $\alpha_{1}=A_{23}, \alpha_{2}=A_{13}^{A_{23}}, \alpha_{3}=A_{124}, \alpha_{4}=A_{34}$. Replacing $\alpha_{2}$ by $\alpha_{1} \alpha_{2} \alpha_{1}^{-1}=A_{13}$ gives an equivalent presentation for $G=\pi_{1}(X(\mathcal{A}))$. We obtain:

$$
\begin{aligned}
G & =G\left(A_{23}, A_{13}, A_{124}, A_{34}\right) \\
& =\left\langle x_{1}, x_{2}, x_{3}, x_{4} \mid x_{1} x_{2} x_{4}=x_{4} x_{1} x_{2}=x_{2} x_{4} x_{1},\left[x_{1}, x_{3}\right]=\left[x_{2}, x_{3}\right]=\left[x_{4}, x_{3}\right]=1\right\rangle .
\end{aligned}
$$

Consequently, $G \cong F_{2} \times \mathbb{Z}^{2}$.

2.6. Fox calculus. Consider a finitely-presented group $G$, with presentation $G=\left\langle x_{1}, \ldots, x_{n} \mid r_{1}, \ldots, r_{m}\right\rangle$. Let $X_{G}$ be the 2-complex modelled on this presentation, $\widetilde{X}_{G}$ its universal cover, and $C_{*}\left(\widetilde{X}_{G}\right)$ its augmented cellular chain complex. Picking as generators for the chain groups the lifts of the cells of $X_{G}$, the complex $C_{*}\left(\widetilde{X}_{G}\right)$ becomes identified with

$$
(\mathbb{Z} G)^{m} \stackrel{J_{G}=\left(\frac{\partial r_{i}}{\partial x_{j}}\right)^{\phi}}{\longrightarrow}(\mathbb{Z} G)^{n} \stackrel{\left(\begin{array}{c}
x_{1}-1 \\
\cdots \\
x_{n}-1
\end{array}\right)}{\longrightarrow} \mathbb{Z} G \stackrel{\epsilon}{\longrightarrow} \mathbb{Z} \rightarrow 0,
$$

where $\epsilon$ is the augmentation map, $\phi: F_{n} \rightarrow G$ is the projection associated to the presentation, and $\frac{\partial}{\partial x_{j}}: \mathbb{Z} F_{n} \rightarrow \mathbb{Z} F_{n}$ are the Fox derivatives, defined by the rules

$$
\frac{\partial 1}{\partial x_{j}}=0, \quad \frac{\partial x_{i}}{\partial x_{j}}=\delta_{i j}, \quad \frac{\partial(u v)}{\partial x_{j}}=\frac{\partial u}{\partial x_{j}} \epsilon(v)+u \frac{\partial v}{\partial x_{j}} .
$$

Now assume that $H_{1}(G) \cong \mathbb{Z}^{n}$. Let ab : $G \rightarrow \mathbb{Z}^{n}$ be the abelianization map, and set $t_{i}=\mathrm{ab}\left(x_{i}\right)$. This choice of generators for $\mathbb{Z}^{n}$ identifies the group ring $\mathbb{Z} \mathbb{Z}^{n}$ with the ring of Laurent polynomials $\Lambda_{n}=\mathbb{Z}\left[t_{1}^{ \pm 1}, \ldots, t_{n}^{ \pm 1}\right]$ The Alexander matrix of $G$ is the $m \times n$ matrix $A_{G}$, with entries in $\Lambda_{n}$, obtained by abelianizing the Fox Jacobian of $G$ (see [22, 3]):

$$
A_{G}=J_{G}^{\mathrm{ab}} .
$$

Let $\psi: \Lambda_{n} \rightarrow \mathbb{Z}\left[\left[\lambda_{1}, \ldots, \lambda_{n}\right]\right]$ be the ring homomorphism given by $\psi\left(t_{i}\right)=1-\lambda_{i}$ and $\psi\left(t_{i}^{-1}\right)=\sum_{q \geq 0} \lambda_{i}^{q}$, and let $\psi^{(h)}$ be its homogeneous part of degree $h$. Since $H_{1}(G) \cong \mathbb{Z}^{n}$, the entries of $A_{G}$ are in the augmentation ideal $\mathfrak{I}=\left(t_{1}-1, \ldots, t_{n}-1\right)$, and so $\psi^{(0)} A_{G}$ is the zero matrix. The linearized Alexander matrix of $G$ is the $m \times n$ matrix

$$
A_{G}^{\operatorname{lin}}=\psi^{(1)} A_{G},
$$

obtained by taking the degree 1 part of the Alexander matrix. Its entries are integral linear forms in $\lambda_{1}, \ldots, \lambda_{n}$. As noted in [54], the coefficient matrix of $A_{G}^{\text {lin }}$ can be interpreted as the augmented Fox Hessian of $G$ :

$$
\left(A_{G}^{\operatorname{lin}}\right)_{i, j}=\sum_{k=1}^{n} \epsilon\left(\frac{\partial^{2} r_{i}}{\partial x_{k} \partial x_{j}}\right) \lambda_{k} .
$$

2.7. Alexander matrices of arrangements. Let $\mathcal{A}$ be an arrangement, with $|\mathcal{A}|=n, \mathcal{L}_{2}(\mathcal{A})=\left\{I_{1}, \ldots, I_{s}\right\}$, and braid monodromy generators $\alpha_{q}=A_{I_{q}}^{\delta_{q}} \in$ $P_{n}$. The Alexander matrix of $G(\mathcal{A})=G\left(\alpha_{1}, \ldots, \alpha_{s}\right)$ can be computed directly from the Gassner representation of the braid monodromy generators, whereas the linearized Alexander matrix can be computed directly from the intersection lattice: 
(a) The Gassner representation of the pure braid group, $\Theta: P_{n} \rightarrow \operatorname{GL}\left(n, \Lambda_{n}\right)$, is defined by

$$
\Theta(\alpha)=\left(\frac{\partial \alpha\left(x_{i}\right)}{\partial x_{j}}\right)^{\mathrm{ab}}
$$

(b) The matrix $A_{G}$ is obtained by stacking the matrices $\Theta\left(\alpha_{1}\right)-\mathrm{id}, \ldots$, $\Theta\left(\alpha_{s}\right)$ - id, and selecting the rows corresponding to $\check{I}_{1}, \ldots, \check{I}_{s}$.

(c) The matrix $A_{G}^{\operatorname{lin}}$ is obtained by stacking the matrices $A_{I_{1}}^{\operatorname{lin}}, \ldots, A_{I_{s}}^{\operatorname{lin}}$, where

$$
\left(A_{I}^{\operatorname{lin}}\right)_{i, j}=\delta_{j, I}\left(\lambda_{i}-\delta_{i, j} \sum_{k \in I} \lambda_{k}\right), \quad \text { for } i \in \check{I} \text { and } j \in[n] .
$$

and $\delta_{j, I}=1$ if $j \in I$, and 0 otherwise.

Example 2.8. For the "near-pencil" of 4 lines in Example 2.5, the Alexander matrix, and its linearization, are as follows:

$$
\begin{aligned}
A & =\left(\begin{array}{cccc}
t_{1}\left(t_{2} t_{4}-1\right) & t_{1}\left(1-t_{1}\right) & 0 & t_{1} t_{2}\left(1-t_{1}\right) \\
1-t_{2} & t_{1} t_{2}\left(t_{4}-1\right)+t_{1}-1 & 0 & t_{1} t_{2}\left(1-t_{2}\right) \\
t_{1}\left(t_{3}-1\right) & 0 & t_{1}\left(1-t_{1}\right) & 0 \\
0 & t_{2}\left(t_{3}-1\right) & t_{2}\left(1-t_{2}\right) & 0 \\
0 & 0 & t_{3}\left(t_{4}-1\right) & t_{3}\left(1-t_{3}\right)
\end{array}\right), \\
A^{\operatorname{lin}} & =\left(\begin{array}{cccc}
-\lambda_{2}-\lambda_{4} & \lambda_{1} & 0 & \lambda_{1} \\
\lambda_{2} & -\lambda_{1}-\lambda_{4} & 0 & \lambda_{2} \\
-\lambda_{3} & 0 & \lambda_{1} & 0 \\
0 & -\lambda_{3} & \lambda_{2} & 0 \\
0 & 0 & -\lambda_{4} & \lambda_{3}
\end{array}\right) .
\end{aligned}
$$

\section{Resonance varieties and characteristic varieties}

3.1. Resonance varieties. Let $G=\left\langle x_{1}, \ldots, x_{n} \mid r_{1}, \ldots, r_{m}\right\rangle$ be a finitelypresented group. Let $H^{*}(G, \mathbb{K})$ be the cohomology ring of $G$, with coefficients in a field $\mathbb{K}$ (with cup-product denoted by $\cdot$ ).

The $d$-th resonance variety of $G$, over the field $\mathbb{K}$, is the set of $\lambda \in H^{1}(G, \mathbb{K})$ for which there exists a subspace $W \in H^{1}(G, \mathbb{K})$ of dimension $d+1$ such that $\mu \cdot \lambda=0$, for all $\mu \in W$. If char $\mathbb{K} \neq 2$, we have $\lambda \cdot \lambda=0$, and thus

$$
R_{d}(G, \mathbb{K})=\left\{\lambda \in H^{1}(G, \mathbb{K}) \mid \operatorname{dim}_{\mathbb{K}} H^{1}\left(H^{*}(G, \mathbb{K}), \cdot \lambda\right) \geq d\right\},
$$

where $\left(H^{*}(G, \mathbb{K}), \cdot \lambda\right)$ is the chain complex with chains the cohomology groups of $G$, and differentials equal to multiplication by $\lambda$.

If $H_{1}(G) \cong \mathbb{Z}^{n}$, then $H^{1}(G, \mathbb{K})$ may be identified with $\mathbb{K}^{n}$. If, moreover, $H_{2}(G)$ is torsion-free, then $R_{d}(G, \mathbb{K})$ may be identified with the $d$-th determinantal variety of the linearized Alexander matrix:

$$
R_{d}(G, \mathbb{K})=\left\{\lambda \in \mathbb{K}^{n} \mid \operatorname{rank}_{\mathbb{K}} A_{G}^{\operatorname{lin}}(\lambda)<n-d\right\},
$$

where $A_{G}^{\operatorname{lin}}(\lambda)$ denotes the linearization of $A_{G}$, evaluated at $\lambda=\left(\lambda_{1}, \ldots, \lambda_{n}\right) \in \mathbb{K}^{n}$, see [54, Theorem 3.9].

The $\mathbb{K}$-resonance varieties form a descending filtration $\mathbb{K}^{n}=R_{0} \supset R_{1} \supset \cdots \supset$ $R_{n-1} \supset R_{n}=\{\mathbf{0}\}$. The ambient type of each term in the filtration depends only on the truncated cohomology ring $H^{\leq 2}(G)$. More precisely, if $H^{\leq 2}\left(G_{1}\right) \cong H^{\leq 2}\left(G_{2}\right)$, there exists a linear automorphism of $\mathbb{K}^{n}$ taking $R_{d}\left(G_{1}, \mathbb{K}\right)$ to $R_{d}\left(G_{2}, \mathbb{K}\right)$. 
3.2. Resonance varieties of arrangements. The resonance varieties of a complex hyperplane arrangement $\mathcal{A}$ were first defined by Falk in $[15]$, as the cohomology jumping loci of the Orlik-Solomon algebra, $A(\mathcal{A})=H^{*}(X(\mathcal{A}), \mathbb{C})$. In particular, for an arrangement $\mathcal{A}$ of $n$ hyperplanes, he defined $R_{d}(\mathcal{A})$ to be the set of $\lambda \in H^{1}(X(\mathcal{A}), \mathbb{C}) \cong \mathbb{C}^{n}$ for which $\operatorname{dim}_{\mathbb{C}} H^{1}(A(\mathcal{A}), \cdot \lambda) \geq d$. As shown in [54], this definition agrees with the one in (3.1), i.e, $R_{d}(\mathcal{A})=R_{d}(G, \mathbb{C})$, where $G=G(\mathcal{A})$.

The (complex) resonance varieties of arrangements are by now very well understood (see the recent surveys by Falk [16] and Yuzvinsky [74] for a thorough treatment). As noted by Falk [15], $R_{1}(\mathcal{A})$ is contained in the hyperplane

$$
\Delta_{n}=\left\{\lambda \in \mathbb{C}^{n} \mid \sum_{i=1}^{n} \lambda_{i}=0\right\} .
$$

As shown in [10], each component of $R_{1}(\mathcal{A})$ is a linear subspace of $\mathbb{C}^{n}$ (see 44, 4, 45. for other proofs and generalizations). In other words, $R_{1}(\mathcal{A})$ is the union of a subspace arrangement in $\mathbb{C}^{n}$. As shown by Libgober and Yuzvinsky in 46], each subspace of $R_{1}(\mathcal{A})$ has dimension at least 2 , two distinct subspaces meet only at $\mathbf{0}$, and $R_{d}(\mathcal{A})$ is the union of those subspaces of dimension greater than $d$.

A purely combinatorial description of $R_{1}(\mathcal{A})$ was given in $[\mathbf{1 5}$, 46]. A partition $\mathrm{P}=\left(\mathrm{p}_{1}|\cdots| \mathrm{p}_{q}\right)$ of $\mathcal{L}_{1}(\mathcal{A})$ is called neighborly if $\left(\left|\mathrm{p}_{j} \cap I\right| \geq|I|-1\right)$ implies $I \subset \mathrm{p}_{j}$, for all $I \in \mathcal{L}_{2}(\mathcal{A})$. To a neighborly partition $\mathrm{P}$, there corresponds an irreducible subvariety of $R_{1}(\mathcal{A})$,

$$
L_{\mathrm{P}}=\Delta_{n} \cap \bigcap_{\left\{I \in \mathcal{L}_{2}(\mathcal{A}) \mid I \not \subset \mathbf{p}_{j}, \forall j\right\}}\left\{\lambda \mid \sum_{i \in I} \lambda_{i}=0\right\} .
$$

Moreover, $\operatorname{dim} L_{\mathrm{P}}>0$ if and only if a certain bilinear form associated to $\mathrm{P}$ is degenerate. Conversely, all components of $R_{1}(\mathcal{A})$ arise from neighborly partitions of sub-arrangements of $\mathcal{A}$.

In particular, for each $I \in \mathcal{L}_{2}(\mathcal{A})$ with $|I| \geq 3$, there is a local component, $L_{I}=$ $\Delta_{n} \cap\left\{\lambda \mid \lambda_{i}=0\right.$ for $\left.i \notin I\right\}$, corresponding to the partition $(I)$ of $\mathcal{A}_{I}=\left\{H_{i} \mid i \in I\right\}$. Note that $\operatorname{dim} L_{I}=|I|-1$, and thus $L_{I} \subset R_{|I|-2}(\mathcal{A})$. At the other extreme, a component of $R_{1}(\mathcal{A})$ that does not correspond to any proper sub-arrangement $\mathcal{A}^{\prime}$ is called essential. The smallest arrangement for which the resonance variety has an essential component (discovered by Falk [15]) is the braid arrangement, $\mathcal{B}$, with defining polynomial $Q_{\mathcal{B}}=x y z(x-y)(x-z)(y-z)$, see Example 10.3 .

The resonance varieties $R_{d}(G, \mathbb{K})$, where $G=G(\mathcal{A})$ is an arrangement group, and $\mathbb{K}$ is a field of positive characteristic, are much less understood. The study of the varieties $R_{d}\left(G, \mathbb{F}_{q}\right)$ was started in [54]. Recall that the resonance variety $R_{d}(\mathcal{A})$ has integral equations, so we may consider its reduction mod $q$. As it turns out, there are arrangements $\mathcal{A}$ for which $R_{d}\left(G, \mathbb{F}_{q}\right)$ does not coincide with $R_{d}(\mathcal{A}) \bmod q$, at certain "exceptional" primes $q$. The two varieties have the same number of local components. On the other hand, $R_{d}\left(G, \mathbb{F}_{q}\right)$ may have non-local components, even though $R_{d}(\mathcal{A}) \bmod q$ has none (see [54] and Examples 10.5 and 10.7 below).

QUESTION 3.3. Let $G=G(\mathcal{A})$ be an arrangement group, and $\mathbb{K}$ a field of positive characteristic. Are all the irreducible components of $R_{d}(G, \mathbb{K})$ linear?

3.4. Characteristic varieties. Let $G$ be a finitely-presented group. For simplicity, assume again that $G$ has torsion-free abelianization. Set $n=b_{1}(G)$, and fix a basis $t_{1}, \ldots, t_{n}$ for $H_{1}(G) \cong \mathbb{Z}^{n}$. Let $\mathbb{K}$ be a field, with multiplicative group of 
units $\mathbb{K}^{*}$, and let $\operatorname{Hom}\left(G, \mathbb{K}^{*}\right)=\operatorname{Hom}\left(H_{1}(G), \mathbb{K}^{*}\right) \cong \mathbb{K}^{* n}$ be the group of $\mathbb{K}$-valued characters of $G$. The $d$-th characteristic variety of $G$, with coefficients in $\mathbb{K}$, is

$$
V_{d}(G, \mathbb{K})=\left\{\mathbf{t} \in \mathbb{K}^{* n} \mid \operatorname{dim}_{\mathbb{K}} H^{1}\left(G, \mathbb{K}_{\mathbf{t}}\right) \geq d\right\},
$$

where $\mathbb{K}_{\mathbf{t}}$ is the $G$-module $\mathbb{K}$ given by the representation $G \stackrel{\text { ab }}{\longrightarrow} \mathbb{Z}^{n} \stackrel{\mathbf{t}}{\rightarrow} \mathbb{K}^{*}$.

The characteristic varieties of $G$ form a descending filtration, $\mathbb{K}^{* n}=V_{0} \supseteq V_{1} \supseteq$ $\cdots \supseteq V_{n-1} \supseteq V_{n}$, which depends only on the isomorphism type of $G$, up to a monomial change of basis in the algebraic torus $\mathbb{K}^{* n}$ (see e.g. [53]).

Define the depth of a character $\mathbf{t}: G \rightarrow \mathbb{K}^{*}$ (relative to the stratification of $\operatorname{Hom}\left(G, \mathbb{K}^{*}\right)=\mathbb{K}^{* n}$ by the characteristic varieties) to be:

$$
\operatorname{depth}_{\mathbb{K}}(\mathbf{t})=\max \left\{d \mid \mathbf{t} \in V_{d}(G, \mathbb{K})\right\} .
$$

With this notation, $V_{d}(G, \mathbb{K}) \backslash V_{d+1}(G, \mathbb{K})=\left\{\mathbf{t} \in \mathbb{K}^{* n} \mid \operatorname{depth}_{K}(\mathbf{t})=d\right\}$.

The characteristic varieties of $G$ may be identified with the determinantal varieties of the Alexander matrix. Indeed, let $A_{G}(\mathbf{t})$ be the matrix $A_{G}$ evaluated at $\mathbf{t} \in \mathbb{K}^{* n}$. For $0 \leq d<n$, we have:

$$
V_{d}(G, \mathbb{K})=\left\{\mathbf{t} \in \mathbb{K}^{* n} \mid \operatorname{rank}_{\mathbb{K}} A_{G}(\mathbf{t})<n-d\right\} .
$$

Proofs of this result can be found in [35, 44] for $\mathbb{K}=\mathbb{C}$, and [55] for arbitrary $\mathbb{K}$. By a well-known result from commutative algebra (see [13, pp. 511-513]) the $d$-th characteristic variety of $G$ is defined by the ideal ann $\left(\bigwedge^{d}\left(\operatorname{coker} A_{G}\right)\right)$, the annihilator of the $d$-th exterior power of the Alexander module of $G$.

3.5. Characteristic varieties of arrangements. For hyperplane arrangement groups, the characteristic varieties are fairly well understood (at least if $\mathbb{K}=\mathbb{C}$ ). This is due, in great part, to the foundational work by Green and Lazarsfeld [28], Simpson [70], and Arapura [1] on the structure of the cohomology support loci for local systems on quasi-projective algebraic varieties. We summarize the present state of knowledge, as follows.

TheOREM 3.6. Let $\mathcal{A}$ be an arrangement of $n$ hyperplanes, with group $G=$ $\pi_{1}(X(\mathcal{A}))$. Let $V_{d}(\mathcal{A})=V_{d}(G, \mathbb{C}), 1 \leq d \leq n$, be the characteristic varieties of the arrangement. Then:

(a) The components of $V_{d}(\mathcal{A})$ are subtori of the character torus $\mathbb{C}^{* n}$, possibly translated by roots of unity, [1. Translated subtori indeed do occur, |71.

(b) The tangent cone at 1 to $V_{d}(\mathcal{A})$ coincides with $R_{d}(\mathcal{A})$, [10, 44, 4, 45].

The components of $V_{d}(\mathcal{A})$ passing through 1 are therefore combinatorially determined. Given a neighborly partition $\mathrm{P}$ of a sub-arrangement $\mathcal{A}^{\prime} \subset \mathcal{A}$, we will denote by $C_{P}$ the corresponding subtorus of $\mathbb{C}^{* n}$, so that $\mathrm{T}_{1} C_{\mathrm{P}}=L_{\mathrm{P}}$. In particular, if $I \in \mathcal{L}_{2}(\mathcal{A})$, we have a local component, $C_{I} \subset V_{|I|-2}(\mathcal{A})$.

It is not known whether the other components of $V_{d}(\mathcal{A})$ are combinatorially determined. The non-Fano arrangement has an isolated point in $V_{2}$, see $1 \mathbf{1 0}$ and Example 10.5. The deleted $\mathrm{B}_{3}$ arrangement has a 1-dimensional component in $V_{1}$ which does not pass through 1, see 71 and Example 10.6 .

We do not know whether Theorem 3.6(a) holds for $V_{d}(G, \mathbb{K})$, if char $\mathbb{K}>0$. Clearly, Theorem 3.6(酑) fails over $\mathbb{K}=\mathbb{F}_{2}$, since $V_{1}\left(G, \mathbb{F}_{2}\right)=\mathbb{F}_{2}^{* n}=\{\mathbf{1}\}$, whereas $R_{1}\left(G, \mathbb{F}_{2}\right)$ contains local components of the form $L_{I} \bmod 2$. It also fails (in a more subtle way) over $\mathbb{K}=\mathbb{F}_{3}$, as in Example 10.7, where $V_{1}\left(G, \mathbb{F}_{3}\right)$ has 8 components (all local), whereas $R_{1}\left(G, \mathbb{F}_{3}\right)$ has 9 components (one non-local). 
Example 3.7. For the near-pencil in Examples 2.5, 2.8, we have:

$$
\begin{aligned}
R_{1}(G, \mathbb{K}) & =\left\{\left(\lambda_{1}, \lambda_{2}, \lambda_{3}, \lambda_{4}\right\} \in \mathbb{K}^{4} \mid \lambda_{1}+\lambda_{2}+\lambda_{4}=0, \lambda_{3}=0\right\}, \\
V_{1}(G, \mathbb{K}) & =\left\{\left(t_{1}, t_{2}, t_{3}, t_{4}\right\} \in \mathbb{K}^{* 4} \mid t_{1} t_{2} t_{4}=1, t_{3}=1\right\} .
\end{aligned}
$$

Notice that $R_{1}=L_{124}$, which is the local component corresponding to the triple point $v_{3}=l_{1} \cap l_{2} \cap l_{4}$. Similarly, $V_{1}=C_{124}$.

\section{Homology of finite-index subgroups and torsion points on varieties}

4.1. Mod $q$ first Betti numbers. Let $G$ be a finitely-presented group. For simplicity, we will again assume that $H_{1}(G)$ is torsion-free, say of rank $n$. For a prime $q$, let $b_{1}^{(q)}(G)=\operatorname{dim}_{\mathbb{F}_{q}} H_{1}\left(G ; \mathbb{F}_{q}\right)$ be the "mod $q$ first Betti number" of $G$. Also let $b_{1}^{(0)}(G)=b_{1}(G)$ be the usual Betti number. Since homology commutes with direct sums, we have $b_{1}^{(q)}(G)=\operatorname{dim}_{\mathbb{K}} H_{1}(G ; \mathbb{K})$, for any field $\mathbb{K}$ of characteristic $q$.

Let $K \leq G$ be a subgroup of finite index $k=|G: K|$. Classically, one computes the homology of $K$ by Shapiro's lemma: $H_{*}(K, \mathbb{Z})=H_{*}(G, \mathbb{Z}[G / K])$. In [22], Fox showed how to compute $H_{1}(K, \mathbb{Z})$ from the invariant factors of $J_{G}^{\sigma}$, the Jacobian matrix of $G$, followed by the permutation representation $\sigma: G \rightarrow \mathrm{GL}(k, \mathbb{Z})$ on the cosets $G / K$. The only disadvantage of Fox's method is that the matrix $J_{G}^{\sigma}$ may be too large for practical computations. The following result from 55 refines Fox's method, computing the mod $q$ first Betti numbers of $K$ from the ranks of much smaller matrices, provided $K$ is a normal subgroup, and $q \nmid k$.

TheOREM $4.2([\mathbf{5 5}])$. Let $G$ be a finitely-presented group, with $H_{1}(G) \cong \mathbb{Z}^{n}$. Let $\gamma: G \rightarrow \Gamma$ be a homomorphism onto a finite group $\Gamma$, with kernel $K_{\gamma}$. Finally, let $\mathbb{K}$ be a field such that $q=\operatorname{char} \mathbb{K}$ does not divide the order of $\Gamma$, and such that $\mathbb{K}$ contains all the roots of unity of order equal to the exponent of $\Gamma$. Then:

$$
b_{1}^{(q)}\left(K_{\gamma}\right)=n+\sum_{\rho \neq \mathbf{1}} n_{\rho}\left(\operatorname{corank} J_{G}^{\rho \circ \gamma}-n_{\rho}\right),
$$

where the sum is over all non-trivial, irreducible representations of $\Gamma$ over the field $\mathbb{K}$, and where $n_{\rho}$ is the degree of such a representation $\rho: \Gamma \rightarrow \operatorname{GL}\left(n_{\rho}, \mathbb{K}\right)$.

Since $\mathbb{C}$ is algebraically closed, and every complex, irreducible representation of a finite abelian group has degree 1, the next corollary follows at once.

Corollary 4.3 (Libgober [44], Sakuma [66], Hironaka [35]). If $\gamma: G \rightarrow \Gamma$ is a homomorphism of $G$ onto a finite abelian group $\Gamma$, then:

$$
b_{1}\left(K_{\gamma}\right)=n+\sum_{\mathbf{1} \neq \rho \in \operatorname{Hom}\left(\Gamma, \mathbb{C}^{*}\right)}\left(\operatorname{corank} J_{G}^{\rho \circ \gamma}-1\right) .
$$

4.4. Betti numbers and torsion points. If $\Gamma$ is a group of prime order $p$, we may reinterpret the formula from Theorem 4.2, in terms of depth of $p$-torsion points on the character variety of $G$ (relative to the stratification by the characteristic varieties). More precisely, let $q=0$, or $q$ a prime, $q \neq p$. Take a field $\mathbb{K}$ of characteristic $q$ which contains all $p$-roots of unity-for example, $\mathbb{K}=\mathbb{C}$, or $\mathbb{K}=\mathbb{F}_{q^{s}}$, where $s=\operatorname{ord}_{p}(q)$ is the least positive integer such that $p \mid\left(q^{s}-1\right)$. Viewing $\Gamma=\mathbb{Z}_{p}$ as the subgroup of $p$-roots of unity in $\mathbb{K}^{*}$, we may identify $\operatorname{Hom}\left(G, \mathbb{Z}_{p}\right)$ with the subgroup of $p$-torsion points on $\operatorname{Hom}\left(G, \mathbb{K}^{*}\right)$. 
Corollary 4.5 (55). If $K=\operatorname{ker}\left(\gamma: G \rightarrow \mathbb{Z}_{p}\right)$, then:

$$
b_{1}^{(q)}(K)=n+(p-1) \operatorname{depth}_{\mathbb{K}}(\gamma)
$$

In view of this Corollary, we are led to define the following invariants of the group $G$. Let

$$
\beta_{p, d}^{(q)}(G)=\#\left\{K \triangleleft G \mid[G: K]=p \text { and } b_{1}^{(q)}(K)=b_{1}^{(q)}(G)+(p-1) d\right\}
$$

be the number of index $p$, normal subgroups of $G$ for which the $\bmod q$ first Betti number jumps by $(p-1) d$, when compared to that of $G$. Note that $\sum_{d \geq 0} \beta_{p, d}^{(q)}(G)=$ $\frac{p^{n}-1}{p-1}$.

Now let

$$
\operatorname{Tors}_{N}\left(\mathbb{K}^{* n}\right)=\left\{\mathbf{t} \in \mathbb{K}^{* n} \mid \mathbf{t}^{N}=\mathbf{1} \text { and } \mathbf{t} \neq \mathbf{1}\right\}
$$

be the set of points on the algebraic torus $\mathbb{K}^{* n}$ of order precisely equal to $N$. For a subset $V \subset \mathbb{K}^{* n}$, put $\operatorname{Tors}_{N}(V)=\operatorname{Tors}_{N}\left(\mathbb{K}^{* n}\right) \cap V$. With these notations, the $\beta$-invariants of $G$ may be computed from the characteristic varieties, as follows.

THEOREM $4.6([\mathbf{5 5}])$. Let $\mathbb{K}$ be a field of characteristic $q \neq p$, containing all the p-roots of unity. Then:

$$
\beta_{p, d}^{(q)}(G)=\frac{\# \operatorname{Tors}_{p}\left(V_{d}(G, \mathbb{K}) \backslash V_{d+1}(G, \mathbb{K})\right)}{p-1} .
$$

An analogous formula holds for $q=p$, with the characteristic varieties replaced by the resonance varieties over the field $\mathbb{F}_{p}$. Let $G=\left\langle x_{1}, \ldots, x_{n} \mid r_{1}, \ldots, r_{m}\right\rangle$ be a finitely-presented group, with $H_{1}(G) \cong \mathbb{Z}^{n}$ and $H_{2}(G)$ torsion-free. Let $G / \gamma_{3} G$ be the second nilpotent quotient of $G$ (see 8.1). The following invariants of $G$ (in fact, of $\left.G / \gamma_{3} G\right)$ were introduced in [54]:

$$
\nu_{p, d}(G)=\#\left\{K \triangleleft G / \gamma_{3} G|| G / \gamma_{3} G: K \mid=p \text { and } b_{1}^{(p)}(K)=n+d\right\} .
$$

We then have:

Theorem $4.7(\mathbf{5 4}) \cdot \nu_{p, d}(G)=\frac{\left|R_{d}\left(G, \mathbb{F}_{p}\right) \backslash R_{d+1}\left(G, \mathbb{F}_{p}\right)\right|}{p-1}$.

\section{Congruence covers and polynomial periodicity}

5.1. Congruence covers. Let $X$ be a finite cell complex (up to homotopy equivalence). Assume $H_{1}(X) \cong \mathbb{Z}^{n}$. Attached to $X$ there is a canonical sequence of congruence covers, $\left\{X_{N}\right\}_{N \in \mathbb{N}}$, defined as follows: $X_{N}$ is the regular $\left(\mathbb{Z}_{N}\right)^{n}$-cover of $X$ determined by the projection

$$
\pi_{1}(X) \stackrel{\mathrm{ab}}{\longrightarrow} H_{1}(X, \mathbb{Z}) \stackrel{\bmod N}{\longrightarrow} H_{1}\left(X ; \mathbb{Z}_{N}\right) .
$$

The sequence of congruence covers depends, up to homotopy, only on $X$. Indeed, a homotopy equivalence $X \stackrel{\simeq}{\rightarrow} Y$ lifts to homotopy equivalences $X_{N} \stackrel{\simeq}{\rightarrow} Y_{N}$, for all $N \geq 1$. Thus, the sequence of Betti numbers $\left\{b_{1}\left(X_{N}\right)\right\}_{N \in \mathbb{N}}$ is a homotopy-type invariant of $X$.

The first Betti numbers of the congruence covers of $X$ can be expressed in terms of the characteristic varieties of $G=\pi_{1}(X)$. Indeed, we may reformulate the results of Libgober, Sakuma, and Hironaka (Corollary 4.3), in this particular case, as follows. 
Theorem 5.2. For every finite cell complex $X$ with $H_{1}(X) \cong \mathbb{Z}^{n}$,

$$
b_{1}\left(X_{N}\right)=n+\sum_{\mathbf{t} \in \operatorname{Tors}_{N}\left(\mathbb{C}^{* n}\right)} \operatorname{depth}_{\mathbb{C}}(\mathbf{t}) .
$$

As a simple example, take $X=\mathbb{C} \backslash\{n$ points $\}$. Then $\pi_{1}(X)=F_{n}$, and $V_{<n}\left(F_{n}, \mathbb{C}\right)=\mathbb{C}^{* n}, V_{n}\left(F_{n}, \mathbb{C}\right)=\{\mathbf{1}\}$. Thus, $b_{1}\left(X_{N}\right)=n+\left(N^{n}-1\right)(n-1)$, which of course can also be seen by computing Euler characteristics.

5.3. Polynomial periodicity. As first noted by Peter Sarnak (who coined the term), the sequence of first Betti numbers of congruence covers of $X$ is polynomially periodic. More precisely:

Theorem 5.4 (Sarnak-Adams [67], Sakuma 66]). For every finite cell complex $X$, the sequence $b_{1}\left(X_{N}\right)$ is polynomially periodic, i.e., there exists an integer $T \geq 1$, and polynomials $P_{1}(x), \ldots, P_{T}(x)$, such that

$$
b_{1}\left(X_{N}\right)=P_{i}(N), \quad \text { if } N \equiv i \bmod T .
$$

We shall call the minimal such $T$ the period of the sequence of Betti numbers. Notice that the polynomials $P_{i}$ and the period $T$ are homotopy-type invariants of $X$. Theorem 5.4 readily follows from Theorem 5.2, by means of a deep result of Laurent [42] (originally conjectured by Serge Lang).

TheOREM 5.5 (Laurent 42]). If $V$ is a subvariety of $\mathbb{C}^{* n}$, then $\operatorname{Tors}_{N}(V)=$ $\bigcup_{i=1}^{v} \operatorname{Tors}_{N}\left(S_{i}\right)$, where $S_{i}$ are subtori of $\mathbb{C}^{* n}$, possibly translated by roots of unity.

Remark 5.6. If $N=p$ is prime, we may express the Betti number $b_{1}\left(X_{p}\right)$ in terms of the $\beta$-invariants of $G=\pi_{1}(X)$ :

$$
b_{1}\left(X_{p}\right)=n+(p-1) \sum_{d \geq 1} d \beta_{p, d}^{(0)}(G) .
$$

Thus, if the $\beta$-invariants are given by different polynomials, for different primes $p$, non-trivial periodicity in the Betti numbers of congruence covers is likely to ensue.

REMARK 5.7. The above analysis can be carried out in the slightly more general context considered by Sakuma [6]. Let $\pi_{A}: H_{1}(X) \rightarrow A$ be a homomorphism from $H_{1}(X) \cong \mathbb{Z}^{n}$ onto a finitely-generated abelian group $A$. There is then a sequence of $\pi_{A}$-congruence covers, $X_{\pi_{A}, N}$, defined by the projection

$$
\pi_{1}(X) \stackrel{\mathrm{ab}}{\longrightarrow} H_{1}(X) \stackrel{\pi_{A}}{\longrightarrow} A \rightarrow A \otimes \mathbb{Z}_{N} .
$$

Unlike the case discussed above (where $A=\mathbb{Z}^{n}$ ) this sequence of covers is not necessarily a homotopy-type invariant of $X$. Nevertheless, the sequence of Betti numbers, $b_{1}\left(X_{\pi_{A}, N}\right)$, is still polynomially periodic, but the polynomials and the period depend also on $\pi_{A}$.

5.8. Congruence covers of arrangements. Let $\mathcal{A}$ be a hyperplane arrangement, with complement $X=X(\mathcal{A})$. Up to homotopy equivalence, $X$ is a finite cell complex. Let $X_{N}=X_{N}(\mathcal{A})$ be its congruence covers. By Theorem 5.4, the sequence $b_{1}\left(X_{N}(\mathcal{A})\right)$ is polynomially periodic. As far as we know, there are no examples in the literature where non-trivial periodicity occurs (i.e., where $T>1$ ). This is probably due to the following observation, which follows immediately from Theorem 3.6. (a) and Theorem 5.2. 
Proposition 5.9. Suppose the intersection of any two components of the characteristic varieties $V_{d}(\mathcal{A})(d \geq 1)$ is the identity. Then $b_{1}\left(X_{N}(\mathcal{A})\right)$ is a polynomial in $N$ (of degree equal to the maximum dimension of any of those components).

If $n \leq 6$, the above condition is satisfied, and so $b_{1}\left(X_{N}(\mathcal{A})\right)$ is a polynomial sequence. If $n \geq 7$, the condition may fail: two components may meet outside the origin, or miss each other altogether. This leads to the following result.

Proposition 5.10. There exist hyperplane arrangements $\mathcal{A}$ for which the sequence $b_{1}\left(X_{N}(\mathcal{A})\right)$ is polynomially periodic, with period $T>1$.

In Section 10, we give several such examples. The simplest one is the non-Fano plane, with $n=7$ (Example 10.5). The Betti numbers $b_{1}\left(X_{N}(\mathcal{A})\right)$ are given by two polynomials, $P_{1}(N)=9 N^{2}-3$ and $P_{2}(N)=9 N^{2}-2$, depending on whether $N$ is even or odd (thus, the period is $T=2$ ). In all the examples, the non-trivial periodicity of the Betti numbers can be traced to the presence of isolated points of order 2 in $V_{2}(\mathcal{A})$. It can also be explained by the dependence of $\beta_{p, d}^{(0)}$ (viewed as a polynomial in $p$ ) on the parity of the prime $p$ (see Remark 5.6).

REMARK 5.11. Similar results may be obtained for the $\pi_{A}$-congruence covers of $X=X(\mathcal{A})$ mentioned in Remark 5.7. One such family of covers was recently considered by Cohen and Orlik [5]. They take $A=\mathbb{Z}$, and $\pi_{A}=(1, \ldots, 1)^{\top}$ : $\mathbb{Z}^{n} \rightarrow \mathbb{Z}$, where $n=|\mathcal{A}|$, and study the resulting sequence of $N$-fold cyclic covers, $X_{(N)}=X_{\pi_{A}, N}$ (the motivation being that, if $X^{*}=X(\mathbf{d} \mathcal{A})$, then $X_{(n)}^{*}$ is the fiber of the Milnor fibration, $\left.Q_{\mathcal{A}}: X(\mathcal{A}) \rightarrow \mathbb{C}^{*}\right)$. Again, non-trivial polynomial periodicity appears in the sequence $b_{1}\left(X_{(N)}\right)$. But the non-trivial periodicity of the sequence of Betti numbers of congruence covers is a much rarer phenomenon than that of cyclic covers. For example, if $X=X(\mathcal{B})$ is the complement of the braid arrangement, the sequence $b_{1}\left(X_{(N)}\right)$ has period $T=3$, whereas $b_{1}\left(X_{N}\right)$ has trivial period $(T=1)$.

\section{Hirzebruch covering surfaces}

6.1. Branched covers. Let $\mathcal{A}=\left\{H_{1}, \ldots, H_{n}\right\}$ be a central arrangement of planes in $\mathbb{C}^{3}$, with complement $X(\mathcal{A})$. Let $\overline{\mathcal{A}}=\left\{\bar{H}_{1}, \ldots, \bar{H}_{n}\right\}$ be the corresponding arrangement of projective lines in $\mathbb{C P}^{2}$, with complement $X(\overline{\mathcal{A}})=\mathbb{C P}^{2} \backslash \bigcup_{i=1}^{n} \bar{H}_{i}$. Notice that $X(\overline{\mathcal{A}})$ is diffeomorphic to the complement of any decone of $\mathcal{A}$. In particular, $H_{1}(X(\overline{\mathcal{A}}))=\mathbb{Z}^{n-1}$.

For each plane arrangement $\mathcal{A}$, and positive integer $N$, Hirzebruch defined in 37 a compact, smooth, complex algebraic surface, $M_{N}(\mathcal{A})$, as follows. Let $X_{N}(\overline{\mathcal{A}})$ be the congruence cover of $X(\overline{\mathcal{A}})$, and let $\widehat{X}_{N}(\overline{\mathcal{A}})$ be the associated branched cover (or, "Kummer cover") of $\mathbb{C P}^{2}$. This is an algebraic surface with normal singularities which ramifies over the projective plane, with the arrangement as the branching locus. By definition, the Hirzebruch covering surface $M_{N}(\mathcal{A})$ is the minimal desingularization of $\widehat{X}_{N}(\overline{\mathcal{A}})$. The resolution of singularities can be described concretely: If a point $z \in \mathbb{C P}^{2}$ lies on $r$ lines of $\overline{\mathcal{A}}$, then the preimage of $z$ in $\widehat{X}_{N}(\overline{\mathcal{A}})$ consists of $N^{n-1-r}$ points, forming an orbit of the Galois group $\left(\mathbb{Z}_{N}\right)^{n-1}$. For $r \geq 3$, each such point $\hat{z}$ is singular. The minimal resolution of $\hat{z}$ replaces it by a smooth curve of genus $2^{r-3}(r-4)+1$ and self-intersection $-2^{r-2}$.

6.2. Chern numbers. In 37, Hirzebruch computed the Chern numbers of $M=M_{N}(\mathcal{A})$, in terms of simple combinatorial data associated to $\mathcal{A}$. The algebraic surface $M$ has Chern numbers $c_{1}^{2}$ (equal to the self-intersection number of 
a canonical divisor) and $c_{2}$ (equal to the Euler characteristic of $M$ ). If $\mathcal{A}_{n}$ is an arrangement for which $\overline{\mathcal{A}}_{n}$ is a pencil of $n$ projective lines, then $c_{1}^{2}$ and $c_{2}$ depend only on $n$ and $N$, see Example 10.1. Otherwise, let $n=|\mathcal{A}|, s=\left|\mathcal{L}_{2}(\mathcal{A})\right|$, and $m_{r}=\#\left\{I \in \mathcal{L}_{2}(\mathcal{A})|| I \mid=r\right\}$. Recall that $\sum_{r} m_{r}(r-1)=b_{2}$, where $b_{2}=b_{2}(X(\mathcal{A}))$.

Theorem 6.3 (Hirzebruch [37]). The Chern numbers of $M_{N}(\mathcal{A})$ are given by

$$
\begin{aligned}
& c_{1}^{2}=\left(\left(3 b_{2}-s-5 n+9\right) N^{2}-4\left(b_{2}-n\right) N+\left(b_{2}+n+m_{2}\right)\right) N^{n-3}, \\
& c_{2}=\left(\left(b_{2}-2 n+3\right) N^{2}-2\left(b_{2}-n\right) N+\left(b_{2}+s-m_{2}\right)\right) N^{n-3} .
\end{aligned}
$$

For example, if $\mathcal{B}$ is the braid arrangement, with $n=6, s=7, m_{2}=3$, and $b_{2}=11$, then $M_{2}(\mathcal{B})$ has $c_{1}^{2}=0$ and $c_{2}=24$ (in fact, as noted by Hirzebruch, $M_{2}(\mathcal{B})$ is the Kummer surface).

6.4. Irregularity. The Betti numbers of the compact algebraic surface $M=$ $M_{N}(\mathcal{A})$ can be computed from $c_{2}=\chi(M)$ by Poincaré duality, once $b_{1}(M)$ is known. The first Betti number $b_{1}(M)$ - equal to twice the irregularity $q(M)$ depends on more subtle data than the Chern numbers. Several methods for computing $b_{1}\left(M_{N}(\mathcal{A})\right)$ were given by Ishida [38], Gläser [26], Zuo 75], and Hironaka 34. For example, Ishida found $b_{1}\left(M_{5}(\mathcal{B})\right)=60$; more generally, Gläser found $b_{1}\left(M_{N}(\mathcal{B})\right)=5(N-1)(N-2)$. Hironaka produced detailed tables of computations of $b_{1}$ (and also $c_{1}^{2}, c_{2}$ ) for surfaces $M_{N}(\mathcal{A})$ with $n \leq 7$ and $N \leq 5$.

In [66], Sakuma gave a new and powerful formula for computing the first Betti numbers of finite abelian branched covers, in a wider context, in terms of nullities of Alexander matrices. We find it convenient to reinterpret Sakuma's formula in terms of depth of complex characters. We do this only for Hirzebruch covering surfaces, though a similar interpretation in a more general context is possible.

Let $x_{1}, \ldots, x_{n}$ be the meridians of $\mathcal{A}$, which recall generate $H_{1}(G(\mathcal{A})) \cong \mathbb{Z}^{n}$. For a character $\mathbf{t} \in \operatorname{Hom}\left(H_{1}(G(\mathcal{A})), \mathbb{C}^{*}\right) \cong \mathbb{C}^{* n}$, and a sub-arrangement $\mathcal{A}^{\prime} \subset \mathcal{A}$, let $\left.\mathbf{t}\right|_{\mathcal{A}^{\prime}}$ be the pull-back of $\mathbf{t}$ to $\operatorname{Hom}\left(H_{1}\left(G\left(\mathcal{A}^{\prime}\right)\right)\right.$, $\left.\mathbb{C}^{*}\right)$, via the obvious inclusion $H_{1}\left(G\left(\mathcal{A}^{\prime}\right)\right) \rightarrow H_{1}(G(\mathcal{A}))$. Also, let $\mathcal{A}_{\mathbf{t}}=\left\{H_{i} \in \mathcal{A} \mid \mathbf{t}\left(x_{i}\right) \neq 1\right\}$ be the sub-arrangement on which $\mathbf{t}$ is supported. Finally, recall that $\operatorname{depth}_{\mathbb{C}}(\mathbf{t})=\max \left\{d \mid \mathbf{t} \in V_{d}(\mathcal{A})\right\}$. With this notation, Sakuma's formula [66] can be written as follows.

TheOREM 6.5. The first Betti number of $M_{N}(\mathcal{A})$ is given by

$$
b_{1}\left(M_{N}(\mathcal{A})\right)=\sum_{\mathbf{t} \in \operatorname{Tors}_{N}\left(\mathbb{C}^{* n}\right)} \operatorname{depth}_{\mathbb{C}}\left(\left.\mathbf{t}\right|_{\mathcal{A}_{\mathbf{t}}}\right) .
$$

For example, the characteristic variety of the braid arrangement, $V_{1}(\mathcal{B})$, has five 2-dimensional components, one of which is non-local, see Example 10.3. Applying Theorem 6.5, one readily recovers Gläser's formula. I]

In 72, Tayama used Sakuma's formula (in its original form) to complete Hironaka's tables of Betti numbers of $M_{N}(\mathcal{A})$, also for $n \leq 7$, but for arbitrary $N$. Moreover, he computed the Betti numbers for pencils of arbitrary size, finding:

$$
b_{1}\left(M_{N}\left(\mathcal{A}_{n}\right)\right):=b(N, n)=(N-1)\left((n-2) N^{n-2}-2 \sum_{k=0}^{n-3} N^{k}\right) .
$$

\footnotetext{
${ }^{4}$ In hindsight, Falk's discovery of the fifth component of $R_{1}(\mathcal{B})$ - the tangent cone at the origin to $V_{1}(\mathcal{B})$ - was predicted 14 years earlier by Ishida and Gläser!
} 
As a consequence, Tayama derived the lower bound

$$
b_{1}\left(M_{N}(\mathcal{A})\right) \geq \sum_{r \geq 3} m_{r} b(N, r)+\beta(\mathcal{A}) b(N, 3),
$$

where $\beta(\mathcal{A})$ is the number of braid sub-arrangements of $\mathcal{A}$.

REMARK 6.6. An inspection of the tables in [72] shows that equality holds in (6.2) for all arrangements $\mathcal{A}$ with $n \leq 7$. This is not surprising, since, in that range, the only sub-arrangement of $\mathcal{A}$ that can have an essential component in its characteristic varieties is the braid arrangement $\mathcal{B}$.

If $n \geq 8$, though, Tayama's inequality can very well be strict, as the arrangements in Examples 10.6, 10.8, 10.9, and 10.11 show. For each such arrangement $\mathcal{A}$, the characteristic varieties have essential components that do not come from braid sub-arrangements, and some of the torsion points on these components add to the Betti number $b_{1}\left(M_{N}(\mathcal{A})\right)$.

6.7. Polynomial periodicity. By Theorem 5.4, the sequence of first Betti numbers of (unramified) congruence covers, $b_{1}\left(X_{N}(\mathcal{A})\right)$, is polynomially periodic. It it by no means evident, but still true, that the same holds for the ramified congruence covers, and their minimal desingularizations.

Theorem 6.8 (Hironaka [33], Sakuma [66]). For every arrangement $\mathcal{A}$, the sequence $b_{1}\left(M_{N}(\mathcal{A})\right)$ is polynomially periodic.

If $n \leq 7$, the sequence $b_{1}\left(M_{N}(\mathcal{A})\right)$ is in fact given by a polynomial in $N$, see Remark 6.6. According to Hironaka [36], the first (and seemingly the only) example of an arrangement for which non-trivial periodicity occurs was given by Zuo in 75. This is the Hessian arrangement, $\mathcal{H}$, with defining polynomial $Q_{\mathcal{H}}=$ $x y z \prod_{i=0}^{2} \prod_{j=0}^{2}\left(x+\omega^{i} y+\omega^{j} z\right)$, where $\omega=e^{2 \pi \mathrm{i} / 3}$. The Betti numbers $b_{1}\left(M_{N}(\mathcal{H})\right)$

are given by two distinct polynomials in $N$, depending on whether $N$ satisfies certain divisibility conditions. Thus, the period $T$ is at least 2 , though the precise value of $T$ does not seem to be known.

The next result shows that periodicity in $b_{1}\left(M_{N}(\mathcal{A})\right)$ occurs for the minimum possible value of $n=|\mathcal{A}|$. Moreover, the period can be identified precisely.

Proposition 6.9. There exists a hyperplane arrangement $\mathcal{A}$ with $|\mathcal{A}|=8$ for which the sequence $b_{1}\left(M_{N}(\mathcal{A})\right)$ is polynomially periodic, with period $T=4$.

The arrangement $\mathcal{A}$ is the deleted $\mathrm{B}_{3}$-arrangement (unlike Zuo's example, this is a complexified real arrangement). Explicit polynomials describing the periodic behavior of $b_{1}\left(M_{N}(\mathcal{A})\right)$ are given in Example 10.6

Another instance of non-trivial periodicity appears in Example 10.11. The two Ziegler arrangements, $\mathcal{A}_{1}$ and $\mathcal{A}_{2}$, have the same multiplicities, and so the complex surfaces $M_{N}\left(\mathcal{A}_{1}\right)$ and $M_{N}\left(\mathcal{A}_{2}\right)$ have the same Chern numbers. Moreover, both sequences $b_{1}\left(M_{N}\left(\mathcal{A}_{i}\right)\right)$ have period 4 , and both start with 0,108 , and 2,110 , but $b_{1}\left(M_{4}\left(\mathcal{A}_{1}\right)\right)=13,932$ and $b_{1}\left(M_{4}\left(\mathcal{A}_{2}\right)\right)=13,930$.

\section{Counting finite-index subgroups}

7.1. Hall formulas. Let $G$ be a finitely-generated group. For each positive integer $k$, let $a_{k}(G)$ be the number of index $k$ subgroups of $G$. As noted by Marshall Hall [29], these numbers can be computed recursively, starting from $a_{1}(G)=1$, 
by means of the formula

$$
a_{k}(G)=\frac{1}{(k-1) !}\left|\operatorname{Hom}\left(G, S_{k}\right)\right|-\sum_{l=1}^{k-1} \frac{1}{(k-l) !}\left|\operatorname{Hom}\left(G, S_{k-l}\right)\right| a_{l}(G),
$$

provided one knows how to count representations from $G$ to the symmetric groups. (See the survey by Lubotzky 47 for an overview of the subject.)

To do this count, it is convenient to introduce the Hall invariants of $G$, so named after Philip Hall, who first considered them in 30 . Let $\Gamma$ be a finite group. The $\Gamma$-Hall invariant of $G$ is the number of surjective representations of $G$ to $\Gamma$, up to automorphisms of $\Gamma$ :

$$
\delta_{\Gamma}(G)=|\operatorname{Epi}(G, \Gamma) / \operatorname{Aut} \Gamma| .
$$

As noted by P. Hall,

$$
|\operatorname{Hom}(G, \Gamma)|=\sum_{H \leq \Gamma}|\operatorname{Aut}(H)| \delta_{\Gamma}(G) .
$$

By (7.1) and (7.3), the computation of $a_{k}(G)$ reduces to the computation of $\delta_{\Gamma}(G)$, for all subgroups $\Gamma \leq S_{k}$. For example, if $H_{1}(G) \cong \mathbb{Z}^{n}$, we have:

$$
\begin{aligned}
& a_{2}=2^{n}-1, \\
& a_{3}=\frac{1}{2}\left(3^{n}-1\right)+3 \delta_{S_{3}}, \\
& a_{4}=\frac{1}{3}\left(2^{n+1}-1\right)\left(2^{n}-1\right)+4\left(\delta_{D_{8}}+\delta_{A_{4}}+\delta_{S_{4}}\right) .
\end{aligned}
$$

7.2. Abelian representations. If $\Gamma$ is finite abelian, an explicit formula for $\delta_{\Gamma}(G)$ was derived in $[\mathbf{5 5}$. For simplicity, we will give this formula just in the case when $G$ has torsion-free abelianization (if $G=F_{n}$, a similar formula was obtained by Kwak, Chun, and Lee in 4 (41).

Write $\Gamma=\prod_{p|| \Gamma \mid} \Gamma_{p}$, where $p$ runs through all the primes dividing the order of $\Gamma$, and $\Gamma_{p}$ is a finite abelian $p$-group. Clearly, $\delta_{\Gamma}(G)=\prod_{p|| \Gamma \mid} \delta_{\Gamma_{p}}(G)$, and so it is enough to compute $\delta_{\Gamma_{p}}(G)$.

THEOREM $7.3(\mathbf{5 5})$. Let $G$ be a finitely-generated group, with $H_{1}(G) \cong \mathbb{Z}^{n}$. If $\Gamma_{p}=\bigoplus_{i=1}^{k} \mathbb{Z}_{p^{\nu_{i}}}$ is an abelian p-group, then:

$$
\delta_{\Gamma_{p}}(G)=\frac{p^{|\nu|(n-1)-2\langle\nu\rangle} \varphi_{n}\left(p^{-1}\right)}{\varphi_{n-k}\left(p^{-1}\right) \prod_{r \geq 1} \varphi_{m_{r}(\nu)}\left(p^{-1}\right)},
$$

where $|\nu|=\sum_{i=1}^{k} \nu_{i},\langle\nu\rangle=\sum_{i=1}^{k}(i-1) \nu_{i}, m_{r}(\nu)=\#\left\{j \mid \nu_{j}=r\right\}$, and $\varphi_{m}(t)=$ $\prod_{i=1}^{m}\left(1-t^{i}\right)$.

7.4. Metabelian representations. The next level of difficulty is presented by finite metabelian groups. We have a formula in that case, too, but only for a special class of (split) metabelian groups, which includes the metacyclic groups considered by Fox in 23. More subtle information about $G$ enters in this formulanot just $n=b_{1}(G)$, but also the stratification of $\operatorname{Hom}\left(G, \mathbb{K}^{*}\right)$ by the characteristic varieties $V_{d}(G, \mathbb{K})$, over certain Galois fields $\mathbb{K}=\mathbb{F}_{q^{s}}$.

For two distinct primes $p$ and $q$, consider the semidirect product $\mathbb{Z}_{q}^{s} \rtimes_{\sigma} \mathbb{Z}_{p}$, where $s=\operatorname{ord}_{p}(q)$ is the order of $q \bmod p$ in $\mathbb{Z}_{p}^{*}$, and $\sigma$ is an automorphism of $\mathbb{Z}_{q}^{s}$ of order $p$. The group $\mathbb{Z}_{q}^{s} \rtimes_{\sigma} \mathbb{Z}_{p}$ is independent of the choice of such $\sigma$. Viewing $\mathbb{Z}_{q}^{s}$ as the additive group of the field $\mathbb{F}_{q^{s}}=\mathbb{F}_{q}(\xi)$, where $\xi$ is a primitive $p$-th root of unity, we may take $\sigma=\cdot \xi \in \operatorname{Aut}\left(\mathbb{F}_{q}(\xi)\right)$. 
TheOREm $7.5([\mathbf{5 5}])$. Let $G$ be a finitely-presented group. Then:

$$
\delta_{\mathbb{Z}_{q}^{s} \rtimes_{\sigma} \mathbb{Z}_{p}}(G)=\frac{p-1}{s\left(q^{s}-1\right)} \sum_{d \geq 1} \beta_{p, d}^{(q)}(G)\left(q^{s d}-1\right) .
$$

The theorem applies to the symmetric group $S_{3}=\mathbb{Z}_{3} \rtimes_{\sigma} \mathbb{Z}_{2}$, where $\sigma=(-1)$, and to the alternating group $A_{4}=\mathbb{Z}_{2}^{2} \rtimes_{\sigma} \mathbb{Z}_{3}$, where $\sigma=\left(\begin{array}{ll}0 & 1 \\ 1 & 1\end{array}\right)$. Combined with Theorem 4.6, it yields:

$$
\begin{aligned}
& \delta_{S_{3}}(G)=\frac{1}{2} \sum_{d \geq 1}\left|\operatorname{Tors}_{2}\left(V_{d}\left(G, \mathbb{F}_{3}\right) \backslash V_{d+1}\left(G, \mathbb{F}_{3}\right)\right)\right|\left(3^{d}-1\right), \\
& \delta_{A_{4}}(G)=\frac{1}{3} \sum_{d \geq 1}\left|\operatorname{Tors}_{3}\left(V_{d}\left(G, \mathbb{F}_{4}\right) \backslash V_{d+1}\left(G, \mathbb{F}_{4}\right)\right)\right|\left(4^{d}-1\right) .
\end{aligned}
$$

Returning now to the low-index subgroups of $G$, we see that $a_{3}(G)$ can be computed from (7.5) and (7.7). As for $a_{4}(G)$, the term $\delta_{A_{4}}$ from (7.6) can be computed from (7.8), but the terms $\delta_{D_{8}}$ and $\delta_{S_{4}}$ cannot be handled by this method.

7.6. Counting normal subgroups. Finally, let $a_{k}^{\triangleleft}(G)$ be the number of index $k$, normal subgroups of $G$. It is readily seen that

$$
a_{k}^{\triangleleft}(G)=\sum_{|\Gamma|=k} \delta_{\Gamma}(G) .
$$

Using Theorem 7.3, we obtain (again, under the assumption $H_{1}(G) \cong \mathbb{Z}^{n}$ ):

$$
\begin{array}{ll}
a_{2}^{\triangleleft}=2^{n}-1, & a_{3}^{\triangleleft}=\frac{1}{2}\left(3^{n}-1\right), \\
a_{4}^{\triangleleft}=\frac{1}{3}\left(2^{n+1}-1\right)\left(2^{n}-1\right), & a_{5}^{\triangleleft}=\frac{1}{4}\left(5^{n}-1\right), \\
a_{6}^{\triangleleft}=\frac{1}{2}\left(3^{n}-1\right)\left(2^{n}-1\right)+\delta_{S_{3}}, & a_{7}^{\triangleleft}=\frac{1}{6}\left(7^{n}-1\right), \\
a_{8}^{\triangleleft}=\frac{1}{21}\left(2^{n+2}-1\right)\left(2^{n+1}-1\right)\left(2^{n}-1\right)+\delta_{D_{8}}+\delta_{Q_{8} .} .
\end{array}
$$

In particular, $a_{k}^{\triangleleft}(G)$ can be computed from the characteristic varieties of $G$, if $k \leq 7$ (more generally, if $k$ has at most two prime factors). This method breaks down at $k=8$, for which $\delta_{D_{8}}$ and $\delta_{Q_{8}}$ are also needed.

\section{Lower central series quotients and Chen groups}

8.1. Lower central series. Let $G$ be a finitely-generated group. If $U_{1}$ and $U_{2}$ are two non-empty subsets of $G$, their commutator subgroup is $\left[U_{1}, U_{2}\right]=\left\{\left[u_{1}, u_{2}\right] \mid\right.$ $\left.u_{1} \in U_{1}, u_{2} \in U_{2}\right\}$, where $\left[u_{1}, u_{2}\right]=u_{1} u_{2} u_{1}^{-1} u_{2}^{-1}$. The commutator subgroup of $G$ is $G^{\prime}=[G, G]$. By repeatedly commuting with $G$, a descending series of fully-invariant subgroups is obtained:

$$
G=\gamma_{1} G \geq \gamma_{2} G \geq \cdots \geq \gamma_{k} G \geq \gamma_{k+1} G \geq \cdots
$$

where $\gamma_{k+1} G=\left[\gamma_{k} G, G\right]$. This is called the lower central series (LCS) of $G$. From the Witt-Hall identities, it follows that

$$
\left[\gamma_{k} G, \gamma_{l} G\right] \leq \gamma_{k+l} G .
$$

(see [49, p. 293]). Let $\operatorname{gr}_{k} G=\gamma_{k} G / \gamma_{k+1} G$ be $k$-th lower central series quotient of $G$. As is well-known, $\operatorname{gr}_{k} G$ is a finitely-generated abelian group (see [49, p. 295]). Let $\phi_{k}(G)=\operatorname{rank}\left(\operatorname{gr}_{k} G\right)$ be its rank. Notice that $\phi_{1}(G)=b_{1}(G)$. 
For the free group $F_{n}$, the LCS quotients are torsion-free, of ranks equal to the Witt numbers:

$$
\phi_{k}\left(F_{n}\right)=w_{k}(n):=\frac{1}{k} \sum_{d \mid k} \mu(d) n^{k / d},
$$

where $\mu$ is the Möbius function, see 49]. By Möbius inversion, $\sum_{d \mid k} d w_{d}(n)=n^{k}$. In particular, $\phi_{1}\left(F_{n}\right)=n$ and $\phi_{p}\left(F_{n}\right)=\frac{n^{p}-n}{p}$, for $p$ prime. Moreover, $\phi_{k}\left(F_{n}\right) \leq n^{k}$.

Now assume $G$ has a presentation with $n$ generators - for example, $G$ is the group of an arrangement of $n$ hyperplanes. The projection $F_{n} \rightarrow G$ induces surjections $\operatorname{gr}_{k} F_{n} \rightarrow \operatorname{gr}_{k} G$. Thus, $\phi_{k}(G) \leq \phi_{k}\left(F_{n}\right) \leq n^{k}$. A sharper upper bound was recently found by Newman, Schneider, and Shalev [57]: If $G \neq F_{n}$, there is an $\epsilon>0$ such that

$$
\phi_{k}(G) \leq(n-\epsilon)^{k}, \quad \text { for } k \text { sufficiently large. }
$$

8.2. Chen Groups. Let $G / G^{\prime \prime}$ be the quotient of $G$ by its second derived subgroup. The group $G / G^{\prime \prime}$ is metabelian (in fact, maximal among all metabelian quotients of $G$ ), and, of course, finitely-generated. It fits into the exact sequence

$$
0 \rightarrow G^{\prime} / G^{\prime \prime} \rightarrow G / G^{\prime \prime} \rightarrow G / G^{\prime} \rightarrow 0 .
$$

The $k$-th Chen group of $G$ is, by definition, $\operatorname{gr}_{k}\left(G / G^{\prime \prime}\right)$, see [56, 50]. Let $\theta_{k}(G)=$ $\phi_{k}\left(G / G^{\prime \prime}\right)$ be its rank.

The projection $G \rightarrow G / G^{\prime \prime}$ induces surjections $\operatorname{gr}_{k} G \rightarrow \operatorname{gr}_{k} G / G^{\prime \prime}$. Thus,

$$
\phi_{k} \geq \theta_{k}, \text { for all } k \text {. }
$$

From (8.2), we have $G^{\prime \prime}=\left[\gamma_{2} G, \gamma_{2} G\right] \leq \gamma_{4} G$, and so $\phi_{k}=\theta_{k}$ for $k \leq 3$. The inequality (8.6) is usually strict for $k>3$. For example (see [56, , 51, 9]):

$$
\theta_{k}\left(F_{n}\right)=\left(\begin{array}{c}
n+k-2 \\
k
\end{array}\right)(k-1), \quad \text { for } k \geq 2,
$$

and this sequence grows much more slowly than the sequence (8.3) of Witt numbers.

Now assume $G / G^{\prime} \cong \mathbb{Z}^{n}$, with generators $t_{1}, \ldots, t_{n}$. The Chen groups of $G$ can be determined from the Alexander invariant $B=G^{\prime} / G^{\prime \prime}$ (viewed as a module over $\left.\mathbb{Z}\left[G / G^{\prime}\right] \cong \mathbb{Z}\left[t_{1}^{ \pm 1}, \ldots, t_{n}^{ \pm 1}\right]\right)$. Indeed, let $\operatorname{gr}_{k} B=\mathfrak{I}^{k} B / \mathfrak{I}^{k+1} B$, where $\mathfrak{I}$ is the augmentation ideal. Then $\operatorname{gr}_{k}\left(G / G^{\prime \prime}\right)=\operatorname{gr}_{k-2} B$, for $k \geq 2$ (see Massey [50]). In particular, we have:

$$
\sum_{k \geq 0} \theta_{k+2} t^{k}=\operatorname{Hilb}(\operatorname{gr} B)
$$

where gr $B=\bigoplus_{k>0} \operatorname{gr}_{k} B$ (viewed as a module over $\operatorname{gr} \mathbb{Z}\left[G / G^{\prime}\right] \cong \mathbb{Z}\left[\lambda_{1}, \ldots, \lambda_{n}\right]$ ). As noted in [6, 9], a presentation for gr $B$ can be obtained from a presentation for $B$ via the well-known Gröbner basis algorithm for finding the tangent cone to a variety (cf. [12]).

8.3. Chen groups of arrangements. Let $\mathcal{A}$ be a complex hyperplane arrangement, with group $G=G(\mathcal{A})$. A finite presentation for the Alexander invariant $B=G^{\prime} / G^{\prime \prime}$ was given in [9]. From this presentation, one can compute the Chen groups of the arrangement, $\operatorname{gr}_{k}\left(G / G^{\prime \prime}\right)$, by the method outlined above (see also Westlund [73] for related work on the Chen groups of the "boundary manifold" of $\mathcal{A})$. 
It seems very likely that the Chen groups of an arrangement are combinatorially determined. We offer a conjecture which makes this combinatorial dependence explicit. First, we need some notation. Let $R_{1}(\mathcal{A})=R_{1}(G, \mathbb{C})$ be the resonance variety of $\mathcal{A}$. Recall that $R_{1}(\mathcal{A})=\bigcup_{i=1}^{v} L_{i}$, with $L_{i}$ linear subspaces of $\mathbb{C}^{n}$, where $n=|\mathcal{A}|$. For each $r \geq 1$, let

$$
h_{r}=\#\left\{L_{i} \mid \operatorname{dim} L_{i}=r\right\}
$$

be the number of components of $R_{1}(\mathcal{A})$ of dimension $r$. Recall also that $m_{r}=$ $\#\left\{I \in \mathcal{L}_{2}(\mathcal{A})|| I \mid=r\right\}$. We know from the discussion in $\S 3.2$ that $h_{1}=0$, and $h_{r} \geq m_{r+1}$ for all $r$. Moreover, the numbers $h_{r}$ can be computed directly from the intersection lattice $\mathcal{L}(\mathcal{A})$, by counting neighborly partitions $\mathrm{P}$ of $\mathcal{A}^{\prime} \subset \mathcal{A}$, and finding $\operatorname{dim} L_{\mathrm{P}}$ by linear algebra.

Conjecture 8.4 (Resonance formula for Chen groups). Let $G=G(\mathcal{A})$ be an arrangement group. Then:

$$
\theta_{k}(G)=\sum_{r \geq 2} h_{r} \theta_{k}\left(F_{r}\right), \quad \text { for } k \geq 4 .
$$

Moreover, the Chen groups $\operatorname{gr}_{k} G / G^{\prime \prime}$ are free abelian (of rank $\theta_{k}$ ), for all $k \geq 1$.

In other words, the conjecture asserts that $\theta_{k}(G)=(k-1) \sum_{r>2} h_{r}\left(\begin{array}{c}k+r-2 \\ k\end{array}\right)$, for $k$ sufficiently large. If we consider only the local components $\left\{L_{I} \mid I \in \mathcal{L}_{2}(\mathcal{A})\right\}$ of $R_{1}(\mathcal{A})$, Conjecture 8.4 implies

$$
\theta_{k}(G) \geq \theta_{k}^{\mathrm{cc}}(G):=(k-1) \sum_{r \geq 3} m_{r}\left(\begin{array}{c}
k+r-3 \\
k
\end{array}\right) .
$$

This inequality is actually known to hold, see [9, Corollary 7.2] for a direct proof.

Conjecture 8.4 grew out of joint work with D. Cohen [6, 9]. An early version of the conjecture appeared in [6]. It stated] $\theta_{k}(G)=\theta_{k}^{\text {cc }}(G)+\beta(\mathcal{A})(k-1)$. Translated into the present language, that earlier conjecture involved only the local components of $R_{1}(\mathcal{A})$, and the non-local components corresponding to braid subarrangements. A counterexample was given in [9]: the Pappus arrangement has an essential component in $R_{1}$, and this adds to the ranks of the Chen groups (see Example 10.9). On the other hand, in all the examples we consider in Section 10 (and many others), Conjecture 8.4 holds.

8.5. LCS quotients of arrangements. If $\mathcal{A}$ is a hyperplane arrangement, with group $G=G(\mathcal{A})$, the LCS ranks $\phi_{k}(G)$ are combinatorially determined. This was proved by Falk in [14], using Sullivan's 1-minimal models. Even so, a precise combinatorial formula for $\phi_{k}$ (even for $\phi_{3}$ ) is not known. Nevertheless, there is a class of arrangements, singled out by Falk and Randell in [17], for which a simple (yet rather deep) formula obtains.

An arrangement $\mathcal{A}=\mathcal{A}_{\ell}$ is called fiber-type if its complement sits atop a tower of linear fibrations,

$$
X=X\left(\mathcal{A}_{\ell}\right) \stackrel{p_{\ell}}{\longrightarrow} X\left(\mathcal{A}_{\ell-1}\right) \rightarrow \cdots \rightarrow X\left(\mathcal{A}_{2}\right) \stackrel{p_{2}}{\longrightarrow} X\left(\mathcal{A}_{1}\right)=\mathbb{C}^{*},
$$

\footnotetext{
${ }^{5}$ Note the similarity with Tayama's lower bound $\left(6.2\right.$ for $b_{1}\left(M_{N}(\mathcal{A})\right)$.
} 
with fibers $p_{i}^{-1}$ (point) $=\mathbb{C} \backslash\left\{d_{i}\right.$ points $\}$. Thus, $X$ is an Eilenberg-MacLane space $K(G, 1)$, with fundamental group

$$
G=F_{d_{\ell}} \rtimes_{\rho_{\ell}} \cdots \rtimes F_{d_{2}} \rtimes_{\rho_{2}} F_{d_{1}} .
$$

From the linearity of the fibrations, it follows that each monodromy map $\rho_{i}$ : $\pi_{1}\left(X\left(\mathcal{A}_{i-1}\right)\right) \rightarrow \operatorname{Aut}\left(F_{d_{i}}\right)$ factors through the pure braid group $P_{d_{i}}$, and thus acts as the identity on homology. A spectral sequence argument shows that the Poincaré polynomial of the complement factors as $P(X, t)=\prod_{i=1}^{\ell}\left(1+d_{i} t\right)$. In particular, the exponents of the fiber-type arrangement, $\left\{d_{1}=1, d_{2}, \ldots, d_{\ell}\right\}$, are combinatorially determined.

The LCS formula of Falk and Randell [17 expresses the lower central series ranks, $\phi_{k}=\phi_{k}(G)$, of a fiber-type arrangement group, $G=G(\mathcal{A})$, in terms of the exponents of $\mathcal{A}$ :

$$
\prod_{k \geq 1}\left(1-t^{k}\right)^{\phi_{k}}=P(X,-t)=\prod_{i=1}^{\ell}\left(1-d_{i} t\right) .
$$

Consequently,

$$
\phi_{k}(G)=\sum_{i=1}^{\ell} w_{k}\left(d_{i}\right) .
$$

As shown by Papadima and Yuzvinsky [61], the converse holds for arrangements in $\mathbb{C}^{3}$ : If $\prod_{k \geq 1}\left(1-t^{k}\right)^{\phi_{k}}=P(X,-t)$, then $\mathcal{A}$ is fiber-type.

The best-known fiber-type arrangement is the braid arrangement $\mathcal{B}_{\ell}$, with exponents $\{1,2, \ldots, \ell-1\}$. In that case, the LCS formula was first proved by Kohno 40. The LCS formula has been an object of intense investigation ever since. It has been interpreted as a consequence of Koszul duality (Shelton-Yuzvinsky 69), and has been generalized to hypersolvable arrangements (Jambu-Papadima [39]), and formal rational $K(G, 1)$ spaces (Papadima-Yuzvinsky [61]).

We propose here a formula for the LCS ranks of a certain class of arrangements. The formula looks very similar to the LCS formula for fiber-type arrangements, with the exponents being replaced by the dimensions of the components of the resonance variety.

ConjeCtURE 8.6 (Resonance LCS formula). Let $G=G(\mathcal{A})$ be an arrangement group. If $\phi_{4}(G)=\theta_{4}(G)$, then:

$$
\phi_{k}(G)=\sum_{r \geq 2} h_{r} \phi_{k}\left(F_{r}\right), \quad \text { for } k \geq 4 .
$$

Moreover, the LCS quotients $\operatorname{gr}_{k} G$ are free abelian (of rank $\phi_{k}$ ), for all $k \geq 1$.

The above formula can also be written as $\phi_{k}(G)=\sum_{r \geq 2} h_{r} w_{k}(r)$, or

$$
\prod_{k \geq 1}\left(1-t^{k}\right)^{\phi_{k}}=\prod_{i=1}^{v}\left(1-\left(\operatorname{dim} L_{i}\right) t\right) .
$$

where recall $R_{1}(\mathcal{A})=\bigcup_{i=1}^{v} L_{i}$ is the decomposition of the resonance variety of $\mathcal{A}$ into (linear) components.

The equality $\phi_{4}=\theta_{4}$ holds for the $\mathrm{X}_{3}, \mathrm{X}_{2}$, Pappus, and non-Pappus arrangements (Examples 10.2, 10.4, 10.9, 10.10). The conjecture is verified in those cases (as well as several others), at least for $k$ up to 8 . 
TABLE 1. Invariants of arrangements

\begin{tabular}{|c|c|}
\hline Defining polynomial & $Q=Q_{\mathcal{A}}$ \\
\hline Multiplicities & $\begin{array}{l}n=\left|\mathcal{L}_{1}(\mathcal{A})\right|, s=\left|\mathcal{L}_{2}(\mathcal{A})\right| \\
m_{r}=\#\left\{I \in \mathcal{L}_{2}(\mathcal{A})|| I \mid=r\right\}\end{array}$ \\
\hline Poincaré polynomial & $\begin{array}{l}P(X, t)=1+b_{1} t+b_{2} t^{2}+b_{3} t^{3} \\
\quad\left(b_{1}=n, b_{2}=\sum_{r} m_{r}(r-1), b_{3}=b_{2}-n+1\right)\end{array}$ \\
\hline Group & $G \cong G^{*} \times \mathbb{Z}$, where $G^{*}=G(\mathbf{d} \mathcal{A})$ \\
\hline Characteristic varieties & $V_{d}(G, \mathbb{K})$, for "generic" $\mathbb{K}$, except when noted \\
\hline Resonance varieties & $R_{d}(G, \mathbb{K})=\mathrm{TC}_{\mathbf{1}}\left(V_{d}(G, \mathbb{K})\right)$, except when noted \\
\hline$\nu$ - and $\beta$-invariants & $\begin{aligned} \nu_{p, d} & =\frac{1}{p-1}\left|R_{d}\left(G, \mathbb{Z}_{p}\right) \backslash R_{d+1}\left(G, \mathbb{Z}_{p}\right)\right| \\
\beta_{p, d}^{(q)} & =\frac{1}{p-1}\left|\operatorname{Tors}_{p}\left(V_{d}(G, \mathbb{K}) \backslash V_{d+1}(G, \mathbb{K})\right)\right| \\
(q & =0, \mathbb{K}=\mathbb{C}, \text { or } q \text { prime }, q \neq p, \mathbb{K}=\mathbb{F}_{\left.q^{\operatorname{ord}_{p}(q)}\right)}\end{aligned}$ \\
\hline Congruence covers & $\begin{array}{l}\text { First Betti numbers } b_{1}\left(X_{N}\right) \text { and } b_{1}\left(M_{N}\right) \\
\text { Chern numbers } c_{1}^{2}\left(M_{N}\right) \text { and } c_{2}\left(M_{N}\right) \\
\end{array}$ \\
\hline $\begin{array}{l}\text { Hall invariants } \\
\text { Low-index subgroups } \\
\end{array}$ & $\begin{array}{l}\delta_{S_{3}}=\frac{1}{2} \sum_{d} \beta_{2, d}^{(3)}\left(3^{d}-1\right), \delta_{A_{4}}=\frac{1}{3} \sum_{d} \beta_{3, d}^{(2)}\left(4^{d}-1\right) \\
a_{2}=2^{n}-1, a_{3}^{\triangleleft}=\frac{1}{2}\left(3^{n}-1\right), a_{3}=a_{3}^{\triangleleft}+3 \delta_{S_{3}}\end{array}$ \\
\hline LCS ranks & $\phi_{1}=n, \phi_{2}=\left(\begin{array}{l}n \\
2\end{array}\right)-b_{2}, \phi_{k}=\operatorname{rankgr}_{k} G$ \\
\hline Chen ranks & $\theta_{1}=\phi_{1}, \theta_{2}=\phi_{2}, \theta_{3}=\phi_{3}, \theta_{k}=\operatorname{rank} \operatorname{gr}_{k}\left(G / G^{\prime \prime}\right)$ \\
\hline
\end{tabular}

*Only non-zero values. For $d=0: \quad \nu_{p, 0}=\frac{p^{n}-1}{p-1}-\sum_{d>0} \nu_{p, d}, \quad \beta_{p, 0}^{(q)}=\frac{p^{n}-1}{p-1}-\sum_{d>0} \beta_{p, d}^{(q)}$.

†Values up to $k=8$. The general term, for $k \geq 4$, is also given, when known from the LCS formula, or, conjecturally, from the Resonance LCS formula.

There are many non-LCS arrangements for which $\phi_{4}>\theta_{4}$, for example, the non-Fano and MacLane arrangements (Examples 10.5, 10.7). In such a situation, the behavior of the sequence $\left\{\phi_{k}\right\}$ is much more complicated (see Peeva 62 for a discussion in the non-Fano case). Furthermore, the LCS quotients may have torsion, as illustrated in Example 10.7. This raises the following question.

QuESTION 8.7. Let $G=G(\mathcal{A})$ be an arrangement group. Is the torsion in $\operatorname{gr}_{k} G$ combinatorially determined?

\section{Guide to examples}

In the next section, we will illustrate the above discussion with a collection of examples of hyperplane arrangements, and some of their associated invariants. In 
TABLE 2. Invariants of arrangements, according to input needed to compute them

\begin{tabular}{l|l}
\hline Multiplicities & $\begin{array}{l}P(X, t), c_{1}^{2}\left(M_{N}\right), c_{2}\left(M_{N}\right), a_{2}, a_{3}^{\triangleleft}, a_{4}^{\triangleleft}, a_{5}^{\triangleleft}, a_{7}^{\triangleleft}, \\
\phi_{1}=\theta_{1}, \phi_{2}=\theta_{2}, \text { and } \phi_{k} \text { (if } \mathcal{A} \text { fiber-type) }\end{array}$ \\
\hline Resonance varieties & $\begin{array}{l}\nu_{p, d}, \theta_{k} \text { (conjecturally), } \\
\phi_{k}\left(\text { conjecturally, if } \phi_{4}=\theta_{4}\right)\end{array}$ \\
\hline Characteristic varieties & $\beta_{p, d}^{(q)}, b_{1}\left(X_{N}\right), b_{1}\left(M_{N}\right), \delta_{S_{3}}, \delta_{A_{4}}, a_{3}, a_{6}^{\triangleleft}$ \\
\hline Other? & $\begin{array}{l}\delta_{\Gamma}(\text { in general }), a_{4}, a_{8}^{\triangleleft}, \phi_{k}(\text { in general }), \\
\text { torsion in } H_{1}\left(X_{N}\right), H_{1}\left(M_{N}\right), \operatorname{gr}_{k}(G)\end{array}$ \\
\hline
\end{tabular}

this section, we provide a guide to those examples, as well as a brief explanation of the computational techniques involved.

In each example, we start with a central arrangement in $\mathbb{C}^{3}$, given by a defining polynomial $Q_{\mathcal{A}}=f_{1} \cdots f_{n}$, with $f_{i}=f_{i}(x, y, z)$ distinct linear forms. If $\mathcal{A}$ is the complexification of a real arrangement, we show a picture of the real part of a decone of $\mathcal{A}$. This is an affine arrangement of lines in $\mathbb{R}^{2}$, with the lines ordered as indicated (the $n$-th line being the "line at infinity"). Otherwise, we merely show the underlying matroid of $\mathcal{A}$, which keeps track of the incidence relations.

Table 1 contains the list of objects and invariants that we associate with $\mathcal{A}$. Some immediate numerical information is extracted from the intersection lattice, $\mathcal{L}(\mathcal{A})$. More refined information is encoded in the fundamental group of the complement, $G=\pi_{1}(X(\mathcal{A}))$, and its resonance and characteristic varieties. Those varieties are depicted schematically, as posets ranked by dimension (indicated on vertical scale), and filtered by depth (indicated by color scheme: solid $=$ component of $V_{1}$, open $=$ component of $V_{2}$, etc). Furthermore, the components of $V_{i}$ and $R_{i}$ are distinguished by their shapes. For example:

$\boldsymbol{\Delta}=$ local component of dimension 2 ,

$\square=$ local component of dimension 3 ,

$\boxplus=$ local component of dimension 4 ,

$\checkmark=$ non-local component from braid sub-arrangement,

$\star=$ non-local component from deleted $\mathrm{B}_{3}$ sub-arrangement, etc.

Table 2 contains the list of invariants under consideration, grouped according to the minimal input needed to compute them. Some of these invariants can be computed from very simple combinatorial data, others from the resonance/characteristic varieties (over various fields), while the remaining ones seem to need another kind of input, either of a very sophisticated combinatorial nature, or altogether noncombinatorial.

Finally, a few words about how the computations were done:

- The package Mathematica 4.0 (from Wolfram Research) was used to find the braid monodromy presentation of the fundamental group, and presentation matrices for various Alexander modules. Also, for standard symbolic computations and organization of information. 
- The algebraic geometry and commutative algebra system Macaulay 2 (by Grayson and Stillman [27]) was used to compute the ideals of minors of various matrices, and their primary decompositions. Also, to compute the tangent cones to modules, and their Hilbert series.

- The group theory package GAP (from the GAP group [24]) was used to manipulate group presentations, find finite-index subgroups, and compute their abelianizations. Also, to compute the lower central series quotients, via the nilpotent quotient algorithm.

\section{Examples}

EXAMPle 10.1 (Central arrangement in $\mathbb{C}^{2}$ ). The most basic arrangement is a pencil of $n$ complex lines in $\mathbb{C}^{2}$. This is a generic 2 -slice of the arrangement $\mathcal{A}_{n}$ mentioned in 5.2 . It is a fiber-type arrangement, with exponents $\{1, n-1\}$.
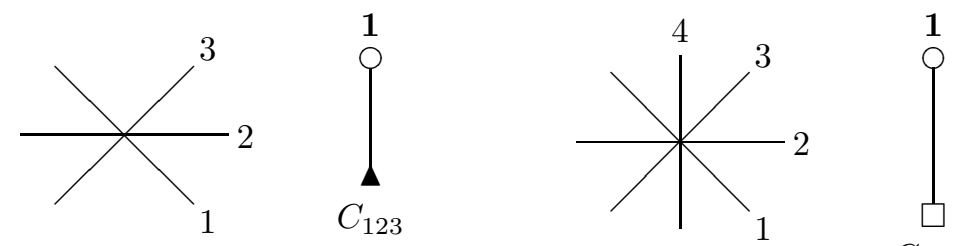

Figure 2. Pencils of 3 and 4 lines

- $Q=(y-x)(x-2 x) \cdots(y-n x)$.

- $s=m_{n}=1$.

- $P(X, t)=(1+t)(1+(n-1) t)$.

- $G^{*}=F_{n-1}, G=F_{n-1} \rtimes_{\alpha} F_{1}$, where $\alpha=A_{12 \ldots n}$.

- $V_{d}(G, \mathbb{K})=\left\{\mathbf{t} \in \mathbb{K}^{* n} \mid t_{1} \cdots t_{n}=1\right\} \cong\left(\mathbb{K}^{*}\right)^{n-1}$, for $d<n-1$ $V_{n-1}(G, \mathbb{K})=\{\mathbf{1}\}$.

- $\beta_{p, n-2}^{(q)}=\nu_{p, n-2}=\frac{p^{n-1}-1}{p-1}$.

- $b_{1}\left(X_{N}\right)=(n-2) N^{n-1}+2$.

- $b_{1}\left(M_{N}\right)=(N-1)\left((n-2) N^{n-2}-2 \sum_{k=0}^{n-3} N^{k}\right)$.

- $c_{1}^{2}\left(M_{N}\right)=N^{n-3}((n-4) N-n)((n-2) N-n)$, $c_{2}\left(M_{N}\right)=2(2-n) N^{n-1}+N^{n-2}+2 n$.

- $\delta_{S_{3}}=\frac{\left(2^{n-1}-1\right)\left(3^{n-2}-1\right)}{2}, \delta_{A_{4}}=\frac{\left(3^{n-1}-1\right)\left(4^{n-2}-1\right)}{6}$.

- $a_{2}=2^{n}-1, a_{3}^{\triangleleft}=\frac{1}{2}\left(3^{n}-1\right), a_{3}=3\left(3^{n-2}-1\right)\left(2^{n-2}+1\right)+4$.

- $\phi_{1}=n, \phi_{2}=\frac{(n-1)(n-2)}{2}, \phi_{3}=\frac{n(n-1)(n-2)}{3}, \phi_{4}=\frac{n(n-1)^{2}(n-2)}{4}$, $\phi_{k}=w_{k}(n-1)$.

- $\theta_{1}=n, \theta_{2}=\frac{(n-1)(n-2)}{2}, \theta_{3}=\frac{n(n-1)(n-2)}{3}, \theta_{4}=\frac{(n+1) n(n-1)(n-2)}{8}$, $\theta_{k}=\left(\begin{array}{c}n+k-3 \\ k\end{array}\right)(k-1)$. 
EXAMPLE 10.2 ( $\mathrm{X}_{3}$ arrangement). This is the smallest arrangement for which the LCS formula does not hold, see Falk and Randell [18].
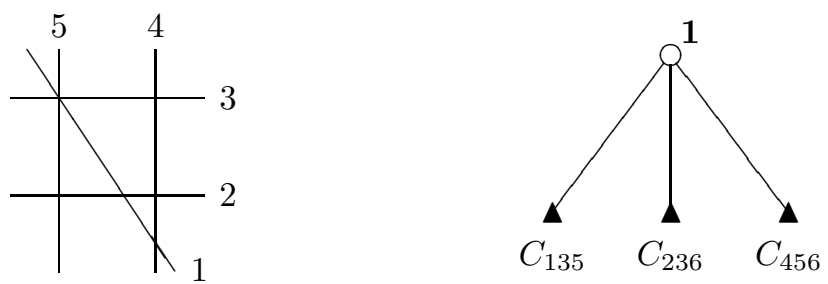

Figure 3. The arrangement $\mathrm{X}_{3}$

- $Q=x y z(y+z)(x-z)(2 x+y)$.

- $n=6, s=9, m_{2}=6, m_{3}=3$.

- $P(X, t)=(1+t)\left(1+5 t+7 t^{2}\right)$.

- $G^{*}=G\left(A_{34}, A_{24}, A_{14}, A_{12}, A_{135}, A_{25}^{A_{35}}\right)$.

- $V_{1}(G, \mathbb{K})=C_{135} \cup C_{236} \cup C_{456}, V_{2}(G, \mathbb{K})=\{\mathbf{1}\}$.

- $\beta_{p, 1}^{(q)}=\nu_{p, 1}=3(p+1)$.

- $b_{1}\left(X_{N}\right)=3\left(N^{2}+1\right)$.

- $b_{1}\left(M_{N}\right)=3(N-1)(N-2)$.

- $c_{1}^{2}\left(M_{N}\right)=6 N^{3}(N-2)^{2}, c_{2}\left(M_{N}\right)=3 N^{3}\left(N^{2}-4 N+5\right)$.

- $\delta_{S_{3}}=9, \delta_{A_{4}}=12$.

- $a_{2}=63, a_{3}^{\triangleleft}=364, a_{3}=391$.

- $\phi_{1}=6, \phi_{2}=3, \phi_{3}=6, \phi_{4}=9, \phi_{5}=18, \phi_{6}=27, \phi_{7}=90, \phi_{8}=150$. Conjecture: $\phi_{k}=3 w_{k}(2)$.

- $\theta_{1}=6, \theta_{2}=3, \theta_{3}=6, \theta_{4}=9, \theta_{k}=3(k-1)$.

EXAMPLE 10.3 (Braid arrangement). This arrangement is a generic 3 -slice of $\mathcal{B}_{4}$. It is a fiber-type arrangement, with exponents $\{1,2,3\}$. The braid arrangement is the smallest arrangement for which the resonance variety contains a non-local component (first found by Falk [15]).

- $Q=x y z(x-y)(x-z)(y-z)$.

- $n=6, s=7, m_{2}=3, m_{3}=4$.

- $P(X, t)=(1+t)(1+2 t)(1+3 t)$.

- $G=P_{4}, G^{*}=\mathbb{F}_{3} \rtimes_{\alpha} \mathbb{F}_{2}$, where $\alpha=\left\{A_{12}, A_{13}\right\}$.

- $V_{1}(G, \mathbb{K})=C_{124} \cup C_{135} \cup C_{236} \cup C_{456} \cup \Pi$, where

$\Pi=C_{(16|25| 34)}=\left\{\left(s, t,(s t)^{-1},(s t)^{-1}, t, s\right) \mid s, t \in \mathbb{K}^{*}\right\}$.

$V_{2}(G, \mathbb{K})=\{\mathbf{1}\}$

- $\beta_{p, 1}^{(q)}=\nu_{p, 1}=5(p+1)$.

- $b_{1}\left(X_{N}\right)=5 N^{2}+1$. 

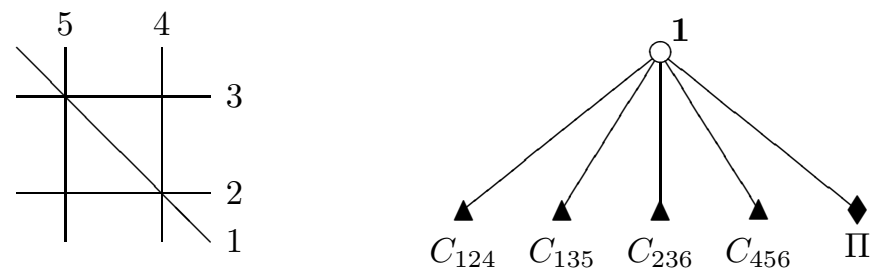

FiguRE 4 . The braid arrangement $\mathcal{B}$

- $b_{1}\left(M_{N}\right)=5(N-1)(N-2)$.

- $c_{1}^{2}\left(M_{N}\right)=5 N^{3}(N-2)^{2}, c_{2}\left(M_{N}\right)=N^{3}\left(2 N^{2}-10 N+15\right)$.

- $\delta_{S_{3}}=15, \delta_{A_{4}}=20$.

- $a_{2}=63, a_{3}^{\triangleleft}=364, a_{3}=409$.

- $\phi_{1}=6, \phi_{2}=4, \phi_{3}=10, \phi_{4}=21, \phi_{k}=w_{k}(2)+w_{k}(3)$.

- $\theta_{1}=6, \theta_{2}=4, \theta_{3}=10, \theta_{4}=15, \theta_{k}=5(k-1)$.

EXAMPLE 10.4 ( $\mathrm{X}_{2}$ arrangement). This is a "parallel" arrangement (first considered by Kohno), for which the LCS formula does not hold, see [61].
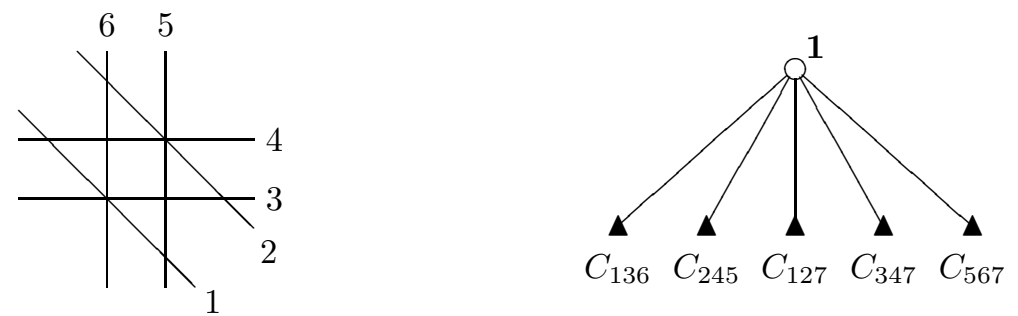

FiguRE 5. The arrangement $\mathrm{X}_{2}$

- $Q=x y z(x+y)(x-z)(y-z)(x+y-2 z)$.

- $n=7, s=11, m_{2}=6, m_{3}=5$.

- $P(X, t)=(1+t)\left(1+6 t+10 t^{2}\right)$.

- $G^{*}=G\left(A_{23}, A_{245}, A_{35}^{A_{45}}, A_{15}^{A_{25} A_{35} A_{45}}, A_{26}, A_{46}, A_{136}^{A_{23} A_{26} A_{46}}, A_{14}^{A_{24}}\right)$.

- $V_{1}(G, \mathbb{K})=C_{136} \cup C_{245} \cup C_{127} \cup C_{347} \cup C_{567}, V_{2}(G, \mathbb{K})=\{\mathbf{1}\}$.

- $\beta_{p, 1}^{(q)}=\nu_{p, 1}=5(p+1)$.

- $b_{1}\left(X_{N}\right)=5 N^{2}+2$.

- $b_{1}\left(M_{N}\right)=5(N-1)(N-2)$.

- $c_{1}^{2}\left(M_{N}\right)=N^{4}\left(11 N^{2}-36 N+29\right), c_{2}\left(M_{N}\right)=N^{4}\left(5 N^{2}-18 N+21\right)$.

- $\delta_{S_{3}}=15, \delta_{A_{4}}=20$.

- $a_{2}=127, a_{3}^{\triangleleft}=1,093, a_{3}=1,138$.

- $\phi_{1}=7, \phi_{2}=5, \phi_{3}=10, \phi_{4}=15, \phi_{5}=30, \phi_{6}=45, \phi_{7}=90, \phi_{8}=150$. Conjecture: $\phi_{k}=5 w_{k}(2)$. 
- $\theta_{1}=7, \theta_{2}=5, \theta_{3}=10, \theta_{4}=15, \theta_{5}=20, \theta_{k}=5(k-1)$.

EXAmPle 10.5 (Non-Fano plane). This is a realization of the celebrated nonFano matroid. It is the smallest arrangement for which one of the characteristic varieties contains a component not passing through the origin (the isolated point $\rho \in V_{2}(G, \mathbb{C})$, first found in $[\mathbf{1 0})$, or for which $R_{d}\left(G, \mathbb{F}_{q}\right)$ does not coincide with $R_{d}(\mathcal{A}) \bmod q$, for some prime $q$ (as noted in [54]).

As we see here, the non-Fano plane is the smallest arrangement for which the congruence covers exhibit periodicity. Moreover, the LCS ranks follow a particularly complicated pattern (the first three were computed by Falk and Randell [17], the next three by Peeva [62], and the seventh is new).

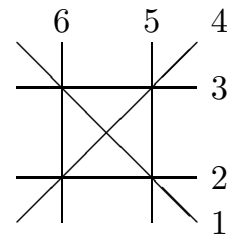

(a) Decone

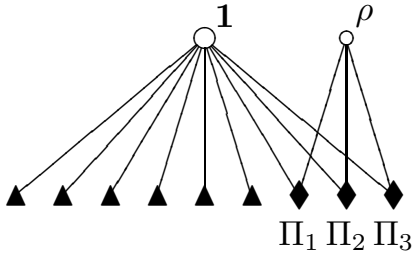

(b) $V_{*}(G, \mathbb{K})$

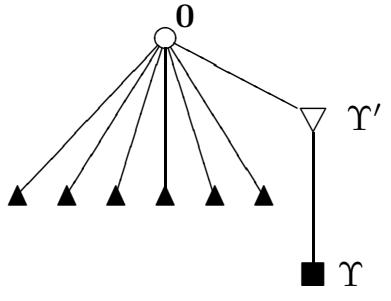

(c) $R_{*}\left(G, \mathbb{F}_{2}\right)$

Figure 6. The non-Fano arrangement

- $Q=x y z(x-y)(x-z)(y-z)(x+y-z)$.

- $n=7, s=9, m_{2}=3, m_{3}=6$.

- $P(X, t)=(1+t)(1+3 t)^{2}$.

- $G^{*}=G\left(A_{345}, A_{125}, A_{14}^{A_{34}}, A_{136}, A_{246}^{A_{34} A_{36}}\right)$.

- $V_{1}(G, \mathbb{K})=C_{125} \cup C_{136} \cup C_{246} \cup C_{345} \cup C_{237} \cup C_{567} \cup \Pi_{1} \cup \Pi_{2} \cup \Pi_{3}$, where $\Pi_{1}=C_{(25|36| 47)}, \Pi_{2}=C_{(17|26| 35)}, \Pi_{3}=C_{(14|23| 56)}$ correspond to braid subarrangements.

$V_{2}(G, \mathbb{K})=\Pi_{1} \cap \Pi_{2} \cap \Pi_{3}=\{\mathbf{1}, \rho\}$, where $\rho=(1,-1,-1,1,-1,-1,1)$, unless char $\mathbb{K}=2$, in which case $V_{2}(G, \mathbb{K})=\{\mathbf{1}\}$.

- $R_{1}\left(G, \mathbb{F}_{2}\right)=L_{125} \cup L_{136} \cup L_{246} \cup L_{345} \cup L_{237} \cup L_{567} \cup \Upsilon$, where

$\Upsilon=L_{(2|3| 5|6| 147)}=\left\{(\mu+\nu, \lambda+\nu, \lambda+\mu+\nu, \nu, \lambda+\mu, \lambda, \mu) \mid \lambda, \mu, \nu \in \mathbb{F}_{2}\right\}$.

$R_{2}\left(G, \mathbb{F}_{2}\right)=\Upsilon^{\prime}$, where $\Upsilon^{\prime}=L_{(2|3| 5 \mid 6)}=\left\{(0, \lambda, \lambda, 0, \lambda, \lambda, 0) \mid \lambda \in \mathbb{F}_{2}\right\}$.

$R_{3}\left(G, \mathbb{F}_{2}\right)=\{\mathbf{0}\}$.

- $\beta_{p, 1}^{(q)}=\nu_{p, 1}=9(p+1)$, except for: $\beta_{2,1}^{(q)}=\nu_{2,1}=24, \beta_{2,2}^{(q)}=\nu_{2,2}=1$.

- $b_{1}\left(X_{N}\right)= \begin{cases}9 N^{2}-3 & \text { if } N \text { even } \\ 9 N^{2}-2 & \text { if } N \text { odd }\end{cases}$

- $b_{1}\left(M_{N}\right)=9(N-1)(N-2)$.

- $c_{1}^{2}\left(M_{N}\right)=N^{4}\left(10 N^{2}-32 N+25\right), c_{2}\left(M_{N}\right)=N^{4}\left(4 N^{2}-16 N+21\right)$.

- $\delta_{S_{3}}=28, \delta_{A_{4}}=36$. 
- $a_{2}=127, a_{3}^{\triangleleft}=1,093, a_{3}=1,177$.

- $\phi_{1}=7, \phi_{2}=6, \phi_{3}=17, \phi_{4}=42, \phi_{5}=123, \phi_{6}=341, \phi_{7}=1,041$.

- $\theta_{1}=7, \theta_{2}=6, \theta_{3}=17, \theta_{4}=27, \theta_{k}=9(k-1)$.

EXAMPLE 10.6 (Deleted $\mathrm{B}_{3}$-arrangement). This arrangement is obtained by deleting a plane from the reflection arrangement of type $B_{3}$ (Example 10.8). It is a fiber-type arrangement, with exponents $\{1,3,4\}$.

The deleted $\mathrm{B}_{3}$-arrangement is the smallest arrangement for which one of the characteristic varieties contains a positive-dimensional component which does not pass through the origin (see $\mathbf{7 1}$ ). As noted in [55], this 1-dimensional translated torus gives rise to 2-torsion in the homology of 3 -fold covers of $X$, and adds 1 to the number of representations of $G$ onto $A_{4}$.

Moreover, as we see here, the translated component in $V_{1}$ (together with the two isolated points in $V_{2}$ ) creates mod 4 periodicity in the sequence of first Betti numbers of Hirzebruch covering surfaces.
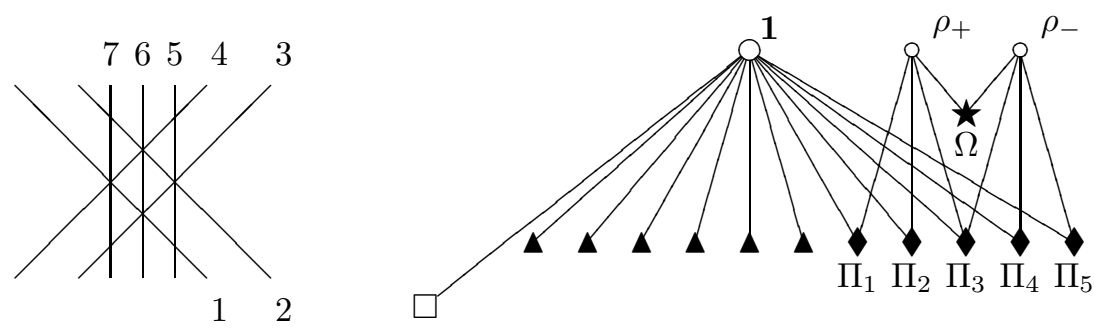

Figure 7 . The deleted $\mathrm{B}_{3}$-arrangement

- $Q=x y z(x-y)(x-z)(y-z)(x-y-z)(x-y+z)$.

- $n=8, s=11, m_{2}=4, m_{3}=6, m_{4}=1$.

- $P(X, t)=(1+t)(1+3 t)(1+4 t)$.

- $G^{*}=\mathbb{F}_{4} \rtimes_{\alpha} \mathbb{F}_{3}$, where $\alpha=\left\{A_{23}, A_{13}^{A_{23}} A_{24}, A_{14}^{A_{24}}\right\}$.

- $V_{1}(G, \mathbb{K})=C_{136} \cup C_{147} \cup C_{235} \cup C_{246} \cup C_{128} \cup C_{348} \cup C_{5678} \cup \bigcup_{i=1}^{5} \Pi_{i} \cup \Omega$, where $\Omega=\left\{\left(t,-t^{-1},-t^{-1}, t, t^{2},-1, t^{-2},-1\right) \mid t \in \mathbb{K}^{*}\right\}$.

$V_{2}(G, \mathbb{K})=C_{5678} \cup\left\{\rho_{+}, \rho_{-}\right\}$, where $\rho_{ \pm}=( \pm 1, \mp 1, \mp 1, \pm 1,1,-1,1,-1)$, unless char $\mathbb{K}=2$, in which case $V_{2}(G, \mathbb{K})=\{\mathbf{1}\}$.

- $\nu_{p, 1}=11(p+1), \nu_{p, 2}=p^{2}+p+1$. $\beta_{p, d}^{(q)}=\nu_{p, d}$, except for: $\beta_{2,1}^{(q)}=27, \beta_{2,2}^{(q)}=9$, and $\beta_{3,1}^{(2)}=45$.

- $b_{1}\left(X_{N}\right)= \begin{cases}2 N^{3}+11 N^{2}+N-9 & \text { if } N \text { even, } \\ 2 N^{3}+11 N^{2}-5 & \text { if } N \text { odd, }\end{cases}$

- $b_{1}\left(M_{N}\right)= \begin{cases}(N-1)\left(2 N^{2}+9 N-24\right)+N-2 & \text { if } N \equiv 0 \bmod 4, \\ (N-1)\left(2 N^{2}+9 N-24\right)+\frac{1}{2}(N-2) & \text { if } N \equiv 2 \bmod 4, \\ (N-1)\left(2 N^{2}+9 N-24\right) & \text { if } N \text { odd }\end{cases}$

- $c_{1}^{2}\left(M_{N}\right)=N^{5}\left(15 N^{2}-44 N+31\right), c_{2}\left(M_{N}\right)=2 N^{5}\left(3 N^{2}-11 N+13\right)$. 
- $\delta_{S_{3}}=63, \delta_{A_{4}}=110$.

- $a_{2}=255, a_{3}^{\triangleleft}=3,280, a_{3}=3,469$.

- $\phi_{1}=8, \phi_{2}=9, \phi_{3}=28, \phi_{4}=78, \phi_{k}=w_{k}(3)+w_{k}(4)$.

- $\theta_{1}=8, \theta_{2}=9, \theta_{3}=28, \theta_{4}=48, \theta_{k}=(k+12)(k-1)$.

EXAMPLE 10.7 (MacLane arrangement). There are two complex realizations of the MacLane matroid $\mathrm{ML}_{8}$ (introduced in 48 ), one for each root of the equation $\omega^{2}+\omega+1=0$. The complements of the two arrangements are diffeomorphic, but not by an orientation-preserving diffeomorphism.

A presentation for the fundamental group $G$ was computed by Rybnikov [65]; the one given here is from [7]. The non-local component in $R_{2}\left(G, \mathbb{F}_{3}\right)$ was first identified in [54. Noteworthy is the presence of torsion in the LCS quotients of $G$.

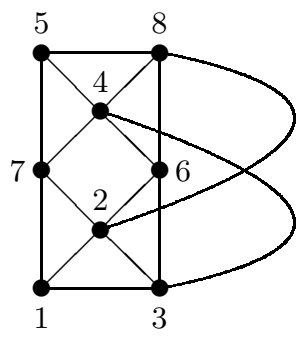

(a) Matroid

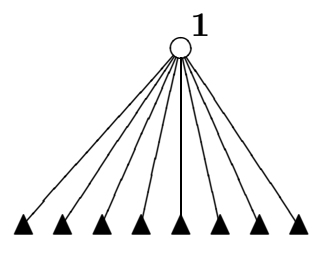

(b) $V_{*}(G, \mathbb{K})$

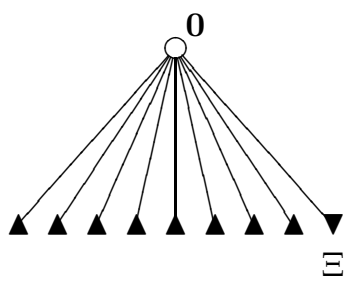

(c) $R_{*}\left(G, \mathbb{F}_{3}\right)$

Figure 8. The MacLane arrangement

- $Q=x y z(y-x)(z-x)(z+\omega y)\left(z+\omega^{2} x+\omega y\right)\left(z-x-\omega^{2} y\right)$.

- $n=8, s=12, m_{2}=4, m_{3}=8$.

- $P(X, t)=(1+t)\left(1+7 t+13 t^{2}\right)$.

- $G^{*}=G\left(A_{456}, A_{36}, A_{126}, A_{134}, A_{25}, A_{47}^{A_{57}}, A_{157}, A_{237}^{A_{57}}\right)$.

- $V_{1}(G, \mathbb{K})=C_{126} \cup C_{134} \cup C_{157} \cup C_{237} \cup C_{258} \cup C_{368} \cup C_{456} \cup C_{478}$, $V_{2}(G, \mathbb{K})=\{\mathbf{1}\}$.

- $R_{1}\left(G, \mathbb{F}_{3}\right)=L_{126} \cup L_{134} \cup L_{157} \cup L_{237} \cup L_{258} \cup L_{368} \cup L_{456} \cup L_{478} \cup \Xi$, where $\Xi=L_{(18|24| 35 \mid 67)}=\left\{(\lambda-\mu, \lambda, \mu,-\lambda,-\mu, \lambda+\mu,-\lambda-\mu, \mu-\lambda) \mid \lambda, \mu \in \mathbb{F}_{3}\right\}$.

- $\beta_{p, 1}^{(q)}=8(p+1)$ and $\nu_{p, 1}=8(p+1)$, except for $\nu_{3,1}=36$.

- $b_{1}\left(X_{N}\right)=8 N^{2}$.

- $b_{1}\left(M_{N}\right)=8(N-1)(N-2)$.

- $c_{1}^{2}\left(M_{N}\right)=N^{5}\left(17 N^{2}-48 N+32\right), c_{2}\left(M_{N}\right)=N^{5}\left(7 N^{2}-24 N+28\right)$.

- $\delta_{S_{3}}=24, \delta_{A_{4}}=32$.

- $a_{2}=255, a_{3}^{\triangleleft}=3,280, a_{3}=3,352$.

- $\phi_{1}=8, \phi_{2}=8, \phi_{3}=21, \phi_{4}=42, \phi_{5}=87, \phi_{6}=105$.

- $\theta_{1}=8, \theta_{2}=8, \theta_{3}=21, \theta_{4}=24, \theta_{k}=8(k-1)$. 
Note. Not all the homology groups of $X_{N}$ are torsion-free. For example, $H_{1}\left(X_{2}\right)=\mathbb{Z}^{32} \oplus \mathbb{Z}_{2}^{4} \oplus \mathbb{Z}_{4}$ and $H_{1}\left(X_{3}\right)=\mathbb{Z}^{72} \oplus \mathbb{Z}_{3}^{8}$.

Note. Not all the LCS quotients of $G$ are torsion-free. For example, $\operatorname{gr}_{5} G=$ $\mathbb{Z}^{87} \oplus \mathbb{Z}_{2}^{4} \oplus \mathbb{Z}_{3}$ and $\operatorname{gr}_{6} G=\mathbb{Z}^{105} \oplus \mathbb{Z}_{2}^{10} \oplus \mathbb{Z}_{4}^{21} \oplus \mathbb{Z}_{16}^{3} \oplus \mathbb{Z}_{3}^{16}$.

EXAMPLE 10.8 ( $\mathrm{B}_{3}$-arrangement). This is the complexification of the reflection arrangement of type $\mathrm{B}_{3}$, consisting of the nine planes of symmetry of the cube in $\mathbb{R}^{3}$, with vertices at $( \pm 1, \pm 1, \pm 1)$. It is a fiber-type arrangement, with exponents $\{1,3,5\}$. The resonance variety has an essential component, first identified in 15.

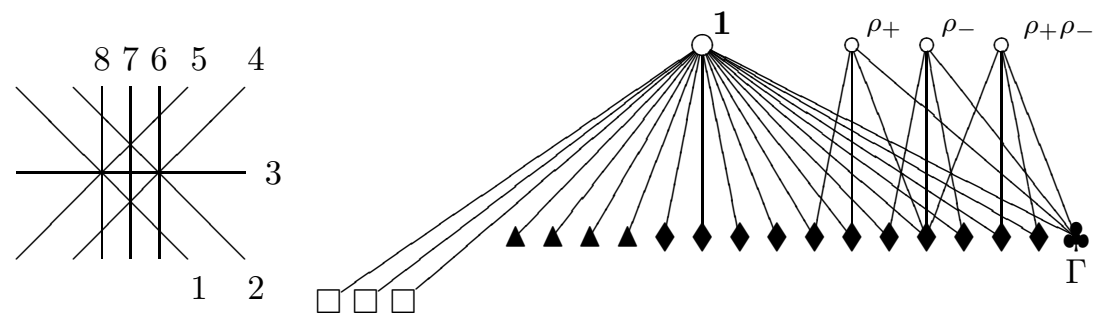

Figure 9. The $\mathrm{B}_{3}$-arrangement

- $Q=x y z(x-y)(x-z)(y-z)(x-y-z)(x-y+z)(x+y-z)$.

- $n=9, s=13, m_{2}=6, m_{3}=4, m_{4}=3$.

- $P(X, t)=(1+t)(1+3 t)(1+5 t)$.

- $G^{*}=\mathbb{F}_{5} \rtimes_{\alpha} \mathbb{F}_{3}$, where $\alpha=\left\{A_{234}, A_{14}^{A_{24} A_{34}} A_{25}, A_{35}^{A_{23} A_{25}}\right\}$.

- $V_{1}(G, \mathbb{K})=C_{147} \cup C_{257} \cup C_{129} \cup C_{459} \cup C_{2346} \cup C_{1358} \cup C_{6789} \cup \bigcup_{i=1}^{11} \Pi_{i} \cup \Gamma$, where $\Gamma=C_{(156|248| 379)}=\left\{\left(t, s,(s t)^{-2}, s, t, t^{2},(s t)^{-1}, s^{2},(s t)^{-1}\right) \mid s, t \in \mathbb{K}^{*}\right\}$. $V_{2}(G, \mathbb{K})=C_{2346} \cup C_{1358} \cup C_{6789} \cup\left\{\rho_{+}, \rho_{-}, \rho_{+} \rho_{-}\right\}$, where $\rho_{ \pm}=( \pm 1, \mp 1,1, \mp 1, \pm 1,1,-1,1,-1)$.

- $\nu_{p, 1}=16(p+1), \nu_{p, 2}=3\left(p^{2}+p+1\right)$. $\beta_{p, d}^{(q)}=\nu_{p, d}$, except for: $\beta_{2,1}^{(q)}=36, \beta_{2,2}^{(q)}=24$.

- $b_{1}\left(X_{N}\right)= \begin{cases}6 N^{3}+16 N^{2}-19 & \text { if } N \text { even } \\ 6 N^{3}+16 N^{2}-13 & \text { if } N \text { odd. }\end{cases}$

- $b_{1}\left(M_{N}\right)=2(N-1)\left(3 N^{2}+5 N-19\right)$.

- $c_{1}^{2}\left(M_{N}\right)=2 N^{6}\left(10 N^{2}-28 N+19\right), c_{2}\left(M_{N}\right)=2 N^{6}\left(4 N^{2}-14 N+15\right)$.

- $\delta_{S_{3}}=132, \delta_{A_{4}}=259$.

- $a_{2}=511, a_{3}^{\triangleleft}=9,841, a_{3}=10,237$.

- $\phi_{1}=9, \phi_{2}=13, \phi_{3}=48, \phi_{4}=168, \phi_{k}=w_{k}(3)+w_{k}(5)$.

- $\theta_{1}=9, \theta_{2}=13, \theta_{3}=48, \theta_{4}=93, \theta_{k}=(3 k+19)(k-1)$.

EXAMPLE 10.9 (Pappus arrangement). This is a realization of the classical $\left(9_{3}\right)_{1}$ configuration of Hilbert and Cohn-Vossen [32]. 

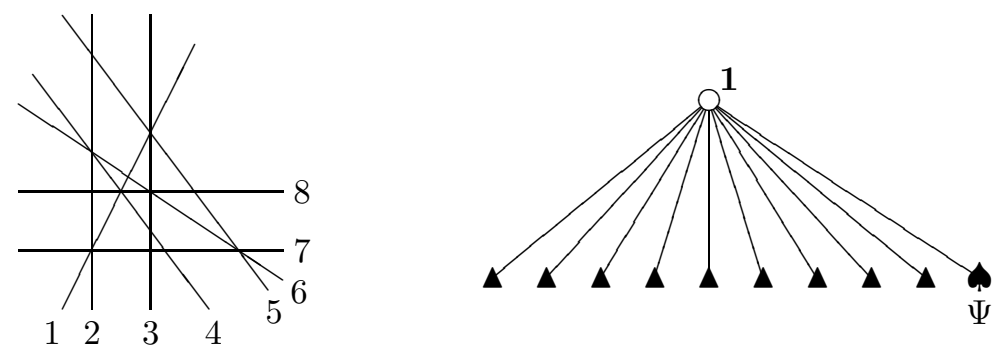

Figure 10. The Pappus arrangement $\left(9_{3}\right)_{1}$

- $Q=x y z(x-y)(y-z)(x-y-z)(2 x+y+z)(2 x+y-z)(2 x-5 y+z)$.

- $n=9, s=18, m_{2}=9, m_{3}=9$.

- $P(X, t)=(1+t)\left(1+8 t+19 t^{2}\right)$.

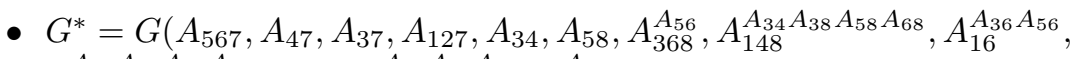
$\left.A_{28}^{A_{38} A_{48} A_{58} A_{68}}, A_{135}, A_{246}^{A_{34} A_{36} A_{56}}, A_{25}^{A_{35}}\right)$.

- $V_{1}(G, \mathbb{K})=C_{567} \cup C_{127} \cup C_{368} \cup C_{148} \cup C_{135} \cup C_{246} \cup C_{239} \cup C_{459} \cup C_{789} \cup \Psi$, where $\Psi=C_{(169|258| 347)}=\left\{\left(t, s,(s t)^{-1},(s t)^{-1}, s, t,(s t)^{-1}, s, t\right) \mid s, t \in \mathbb{K}^{*}\right\}$. $V_{2}(G, \mathbb{K})=\{\mathbf{1}\}$.

- $\beta_{p, 1}^{(q)}=\nu_{p, 1}=10(p+1)$.

- $b_{1}\left(X_{N}\right)=10 N^{2}-1$.

- $b_{1}\left(M_{N}\right)=10(N-1)(N-2)$.

- $c_{1}^{2}\left(M_{N}\right)=9 N^{6}(N-1)(3 N-5), c_{2}\left(M_{N}\right)=12 N^{6}\left(N^{2}-3 N+3\right)$.

- $\delta_{S_{3}}=30, \delta_{A_{4}}=40$.

- $a_{2}=511, a_{3}^{\triangleleft}=9,841, a_{3}=9,931$.

- $\phi_{1}=9, \phi_{2}=9, \phi_{3}=20, \phi_{4}=30, \phi_{5}=60, \phi_{6}=90, \phi_{7}=180, \phi_{8}=300$. Conjecture: $\phi_{k}=10 w_{k}(2)$.

- $\theta_{1}=9, \theta_{2}=9, \theta_{3}=20, \theta_{4}=30, \theta_{5}=50, \theta_{k}=10(k-1)$

ExAmPle 10.10 (Non-Pappus arrangement). This is a realization of the $\left(9_{3}\right)_{2}$ configuration from [32]. It has the same number of multiple points as the Pappus arrangement, but the difference in their position is reflected in several invariants of the complement.
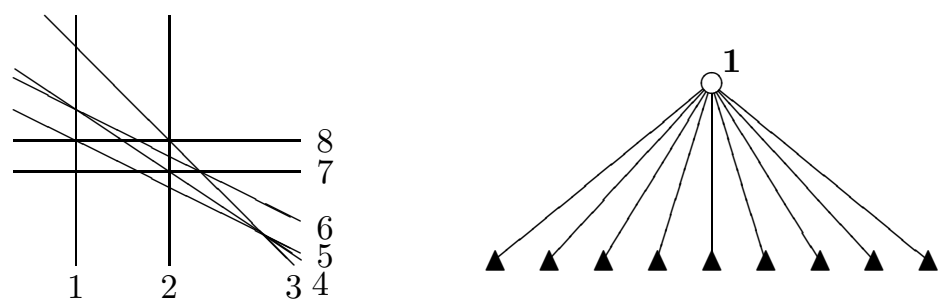

FIgURE 11. The non-Pappus arrangement $\left(9_{3}\right)_{2}$ 
- $Q=x y z(x+y)(y+z)(x+3 z)(x+2 y+z)(x+2 y+3 z)(2 x+3 y+3 z)$.

- $n=9, s=18, m_{2}=9, m_{3}=9$.

- $P(X, t)=(1+t)\left(1+8 t+19 t^{2}\right)$.

- $G^{*}=G\left(A_{345}, A_{367}, A_{25}, A_{247}^{A_{34} A_{36}^{-1}}, A_{26}^{A_{36}}, A_{238}, A_{57}^{A_{67}}, A_{68}, A_{48}, A_{17}^{A_{27} A_{37} A_{47} A_{67}}\right.$, $\left.A_{158}, A_{146}^{A_{24} A_{34} A_{36}}, A_{13}^{A_{23}}\right)$.

- $V_{1}(G, \mathbb{K})=C_{345} \cup C_{367} \cup C_{247} \cup C_{238} \cup C_{158} \cup C_{146} \cup C_{129} \cup C_{569} \cup C_{789}$, $V_{2}(G, \mathbb{K})=\{\mathbf{1}\}$.

- $\beta_{p, 1}^{(q)}=\nu_{p, 1}=9(p+1)$.

- $b_{1}\left(X_{N}\right)=9 N^{2}$.

- $b_{1}\left(M_{N}\right)=9(N-1)(N-2)$.

- $c_{1}^{2}\left(M_{N}\right)=9 N^{6}(N-1)(3 N-5), c_{2}\left(M_{N}\right)=12 N^{6}\left(N^{2}-3 N+3\right)$.

- $\delta_{S_{3}}=27, \delta_{A_{4}}=36$.

- $a_{2}=511, a_{3}^{\triangleleft}=9,841, a_{3}=9,922$.

- $\phi_{1}=9, \phi_{2}=9, \phi_{3}=18, \phi_{4}=27, \phi_{5}=54, \phi_{6}=81, \phi_{7}=162, \phi_{8}=270$. Conjecture: $\phi_{k}=9 w_{k}(2)$.

- $\theta_{1}=9, \theta_{2}=9, \theta_{3}=18, \theta_{4}=27, \theta_{5}=36, \theta_{k}=9(k-1)$.

EXAMPLE 10.11 (Ziegler arrangements). These arrangements, $\mathcal{A}_{1}$ and $\mathcal{A}_{2}$, are both fiber-type, with exponents $\{1,6,6\}$. They are combinatorially very close, yet a subtle difference in the respective lattices gets detected by some of the more refined topological invariants of the complements, such as the number, $\tau$, of translated components in the characteristic varieties [71], or the Hall invariant $\delta_{A_{4}}$. Furthermore, the sequences $b_{1}\left(X_{N}\left(\mathcal{A}_{i}\right)\right)$ and $b_{1}\left(M_{N}\left(\mathcal{A}_{i}\right)\right)$ are polynomially periodic (of period 2 and 4 , respectively), but the corresponding polynomials differ for $N$ even.
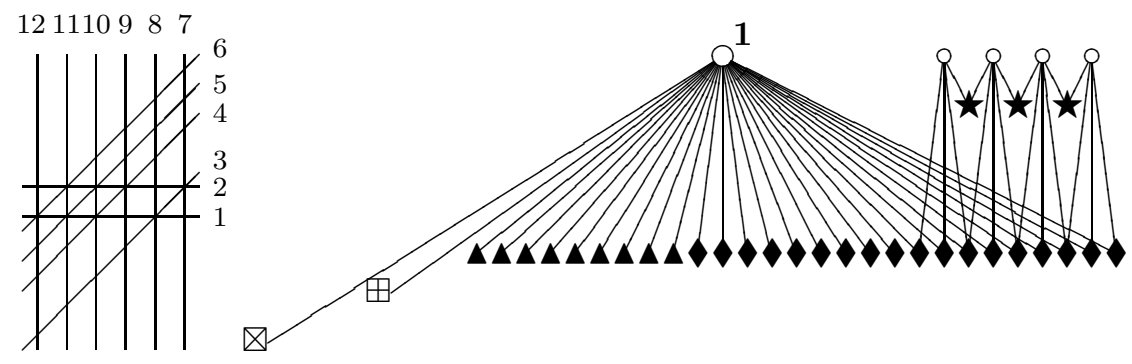

FIGURE 12. The first Ziegler arrangement

- $Q_{\mathcal{A}_{1}}=x y z(x-y)(y-z)(x-z)(x-2 z)(x-3 z)(x-4 z)(x-5 z) \times$ $(x-y-z)(x-y-2 z)(x-y-4 z)$,

$Q_{\mathcal{A}_{2}}=x y z(x-y)(y-z)(x-z)(x-2 z)(x-3 z)(x-4 z)(x-5 z) \times$

$(x-y-z)(x-y-3 z)(x-y-4 z)$.

- $n=13, s=31, m_{2}=20, m_{3}=9, m_{5}=1, m_{7}=1$.

- $P\left(X_{i}, t\right)=(1+t)(1+6 t)^{2}$. 

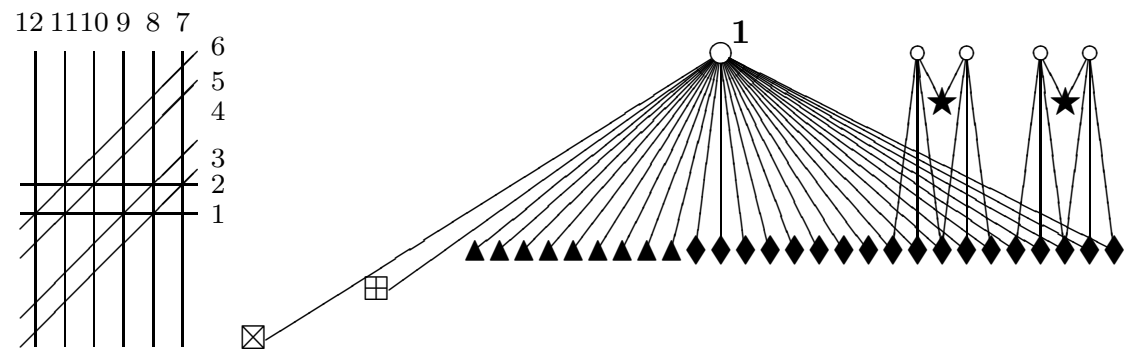

Figure 13. The second Ziegler arrangement

- $G_{i}^{*}=F_{6} \rtimes_{\alpha_{i}} F_{6}$, where $\alpha_{1}=\left\{A_{23}, A_{13}^{A_{23}}, A_{24}, A_{14}^{A_{24}} A_{25}, A_{15}^{A_{25}} A_{26}, A_{16}^{A_{26}}\right\}$, $\alpha_{2}=\left\{A_{23}, A_{13}^{A_{23}} A_{24}, A_{14}^{A_{24}}, A_{25}, A_{15}^{A_{25}} A_{26}, A_{16}^{A_{26}}\right\}$.

- Each variety $V_{1}\left(G_{i}, \mathbb{K}\right)$ has 11 local components, and 18 components corresponding to braid sub-arrangements. In addition, $V_{1}\left(G_{1}, \mathbb{K}\right)$ has $\tau_{1}=3$ components corresponding to deleted $\mathrm{B}_{3}$ sub-arrangements, whereas $V_{1}\left(G_{2}, \mathbb{K}\right)$ has $\tau_{2}=2$ such components (not passing through the origin).

$V_{2}\left(G_{i}, \mathbb{K}\right)=\left\{\mathbf{1}, \rho_{1}, \rho_{2}, \rho_{3}, \rho_{4}\right\}$, with $\rho_{j}^{2}=\mathbf{1} ; V_{2}\left(G_{i}, \mathbb{K}\right)=\{\mathbf{1}\}$, if char $\mathbb{K}=2$.

- $\nu_{p, 1}=27(p+1), \nu_{p, 3}=\frac{p^{4}-1}{p-1}, \nu_{p, 5}=\frac{p^{6}-1}{p-1}$.

$\beta_{p, d}^{(q)}=\nu_{p, d}$, except for $\beta_{2,1}^{(q)}=69, \beta_{2,2}^{(q)}=4$, and

$$
\beta_{3,1}^{(2)}\left(G_{1}\right)=111, \quad \beta_{3,1}^{(2)}\left(G_{2}\right)=110 .
$$

- $b_{1}\left(X_{N}\left(\mathcal{A}_{i}\right)\right)= \begin{cases}5 N^{6}+3 N^{4}+27 N^{2}+\tau_{i}(N-2)-26 & \text { if } N \text { even, } \\ 5 N^{6}+3 N^{4}+27 N^{2}-22 & \text { if } N \text { odd. }\end{cases}$

- $b_{1}\left(M_{N}\left(\mathcal{A}_{i}\right)\right)= \begin{cases}f(N)+\tau_{i}(N-2) & \text { if } N \equiv 0 \bmod 4, \\ f(N)+\frac{\tau_{i}}{2}(N-2) & \text { if } N \equiv 2 \bmod 4, \\ f(N) & \text { if } N \text { odd, }\end{cases}$

where $f(N)=(N-1)\left(5 N^{5}-2 N^{4}+N^{3}-4 N^{2}+23 N-58\right)$.

- $c_{1}^{2}\left(M_{N}\left(\mathcal{A}_{i}\right)\right)=N^{10}\left(57 N^{2}-140 N+81\right)$, $c_{2}\left(M_{N}\left(\mathcal{A}_{i}\right)\right)=N^{10}\left(25 N^{2}-70 N+59\right)$.

- $\delta_{S_{3}}=7,903$, and

$$
\delta_{A_{4}}\left(G_{1}\right)=124,435, \quad \delta_{A_{4}}\left(G_{2}\right)=124,434 .
$$

- $a_{2}=8,191, a_{3}^{\triangleleft}=797,161, a_{3}=820,870$.

- $\phi_{1}=13, \phi_{2}=30, \phi_{3}=140, \phi_{4}=630, \phi_{k}=2 w_{k}(6)$.

- $\theta_{1}=13, \theta_{2}=30, \theta_{3}=140, \theta_{4}=336, \theta_{k}=\frac{(k-1)\left(k^{4}+10 k^{3}+47 k^{2}+86 k+696\right)}{24}$.

\section{References}

[1] D. Arapura, Geometry of cohomology support loci for local systems I, J. Alg. Geom. 6 (1997), 563-597.

[2] W. Arvola, The fundamental group of the complement of an arrangement of complex hyperplanes, Topology 31 (1992), 757-766. 
[3] J. Birman, Braids, links and mapping class groups, Ann. of Math. Studies, vol. 82, Princeton Univ. Press, Princeton, NJ, 1975.

[4] D. Cohen, P. Orlik, Arrangements and local systems, Math. Res. Lett. 7 (2000), 299-316.

[5] Some cyclic covers of complements of arrangements, Topology Appl. (to appear), arXiv:math.AG/0001167.

[6] D. Cohen, A. Suciu, The Chen groups of the pure braid group, In: The Cech Centennial: A Conference on Homotopy Theory (M. Cenkl, H. Miller, eds.), Contemp. Math., vol. 181, Amer. Math. Soc., Providence, RI, 1995, pp. 45-64.

[7] - The braid monodromy of plane algebraic curves and hyperplane arrangements, Comment. Math. Helvetici 72 (1997), 285-315.

[8] - Homology of iterated semidirect products of free groups, J. Pure Appl. Algebra 126 (1998), 87-120.

[9] - Alexander invariants of complex hyperplane arrangements, Trans. Amer. Math. Soc. 351 (1999), 4043-4067.

[10] Characteristic varieties of arrangements, Math. Proc. Cambridge Phil. Soc. 127 (1999), 33-53.

[11] R. Cordovil, J. Fachada, Braid monodromy groups of wiring diagrams, Boll. Unione Mat. Ital. 9 (1995), 399-416.

[12] D. Cox, J. Little, D. O'Shea, Ideals, varieties, and algorithms, 2nd ed., Undergrad. Texts Math., Springer-Verlag, New York, 1997.

[13] D. Eisenbud, Commutative algebra with a view towards algebraic geometry, Grad. Texts in Math., vol. 150, Springer-Verlag, New York, 1995.

[14] M. Falk, The minimal model of the complement of an arrangement of hyperplanes, Trans. Amer. Math. Soc. 309 (1988), 543-556.

[15] _ Arrangements and cohomology, Ann. Combin. 1 (1997), 135-157.

[16] - Combinatorial and algebraic structure in Orlik-Solomon algebras, European J. Combinatorics (to appear), arXiv:math.C0/0009135.

[17] M. Falk, R. Randell, The lower central series of a fiber-type arrangement, Invent. Math. 82 (1985), 77-88.

[18] _ On the homotopy theory of arrangements, In: Complex analytic singularities, Adv. Stud. Pure Math., vol. 8, Math. Soc. Japan, Tokyo, 1986, pp. 101-124.

[19] - Pure braid groups and products of free groups, In: Braids (J. Birman, A. Libgober, eds.), Contemp. Math., vol . 78, Amer. Math. Soc., Providence, RI, 1988, pp. 217-228.

[20] - On the homotopy theory of arrangements, II, In: Arrangements-Tokyo 1998 (M. Falk, H. Terao, eds.), Adv. Stud. Pure Math., vol. 27, Math. Soc. Japan, Kinokuniya, Tokyo, 2000, pp. 93-125.

[21] M. Farber, S. Tabachnikov, Topology of cyclic configuration spaces and periodic trajectories of multi-dimensional billiards, arXiv:math.DG/9911226.

[22] R. H. Fox, Free differential calculus. III. Subgroups, Ann. of Math. 64 (1956), 407-419.

[23] _ Metacyclic invariants of knots and links, Canad. J. Math. 22 (1970), 193-201.

[24] The GAP Group, GAP-Groups. Algorithms, and Programming Version 4.2, Aachen, St. Andrews, 1999; available at http://www-gap.dcs.st-and.ac.uk/ gap.

[25] R. Ghrist, Configuration spaces and braid groups on graphs in robotics, arXiv:math.GT/ 9905023.

[26] M. Gläser, Arbeitsbericht Sonderforschungsbereich, vol. 40, Bonn 1983 (unpublished).

[27] D. Grayson, M. Stillman, Macaulay 2: a software system for algebraic geometry and commutative algebra; available at http://www.math.uiuc.edu/Macaulay2.

[28] M. Green, R. Lazarsfeld, Higher obstructions to deforming cohomology groups of line bundles, J. Amer. Math. Soc. 4 (1991), 87-103.

[29] M. Hall, Subgroups of finite index in free groups, Canad. J. Math 1 (1949), 187-190.

[30] P. Hall, The Eulerian functions of a group, Quart. J. Math 7 (1936), 134-151.

[31] H. Hamm, Lê D. T., Un théorème de Zariski du type de Lefschetz, Ann. Sci. École Norm. Sup. 6 (1973), 317-366.

[32] D. Hilbert, S. Cohn-Vossen, Geometry and the imagination, Chelsea, New York, 1933.

[33] E. Hironaka, Polynomial periodicity for Betti numbers of covering surfaces, Inv. Math. 108 (1992), 289-321.

[34] Abelian coverings of the complex projective plane branched along configurations of real lines, Memoirs A.M.S., vol. 502, Amer. Math. Soc., Providence, RI, 1993. 
[35] - Alexander stratifications of character varieties, Annales de l'Institut Fourier (Grenoble) 47 (1997), 555-583.

[36] _ Multi-polynomial invariants for plane algebraic curves, In: Singularities and complex geometry (Beijing, 1994), AMS/IP Stud. Adv. Math., vol. 5, Amer. Math. Soc., Providence, RI, 1997, pp. 67-74.

[37] F. Hirzebruch, Arrangements of lines and algebraic surfaces, In: Arithmetic and Geometry, vol. II, Progress in Math., vol. 36, Birkhäuser, Boston, 1983, pp. 113-140.

[38] M.-N. Ishida, The irregularities of Hirzebruch's examples of surfaces of general type with $c_{1}^{2}=3 c_{2}$, Math. Ann. 262 (1983), 407-420.

[39] M. Jambu, S. Papadima, A generalization of fiber-type arrangements and a new deformation method, Topology 37 (1998), 1135-1164.

[40] T. Kohno, Série de Poincaré-Koszul associée aux groupes de tresses pures, Invent. Math. 82 (1985), 57-75.

[41] J. H. Kwak, J.-H Chun, J. Lee, Enumeration of regular graph coverings having finite abelian covering transformation groups, SIAM J. Discrete Math. 11 (1998), 273-285.

[42] M. Laurent, Équations diophantiennes exponentielles, Invent. Math. 78 (1984), 299-327.

[43] A. Libgober, On the homology of finite abelian coverings, Topology Appl. 43 (1992), 157166 .

[44] - Characteristic varieties of algebraic curves, arXiv:math.AG/9801070.

[45] - First order deformations for rank one local systems with non vanishing cohomology, Topology Appl. (to appear).

[46] A. Libgober, S. Yuzvinsky, Cohomology of the Orlik-Solomon algebras and local systems, Compositio Math. 21 (2000), 337-361.

[47] A. Lubotzky, Counting finite index subgroups, In: Groups '93, Galway/St. Andrews, Vol. 2, London Math. Soc. Lecture Note Ser., vol. 212, Cambridge Univ. Press, Cambridge, 1995, pp. 368-404.

[48] S. MacLane, Some interpretations of abstract linear independence in terms of projective geometry, Amer. J. Math. 58 (1936), 236-241.

[49] W. Magnus, A. Karrass, D. Solitar, Combinatorial group theory (2nd ed.), Dover, New York, 1976.

[50] W. Massey, Completion of link modules, Duke Math. J. 47 (1980), 399-420.

[51] W. Massey, L. Traldi, On a conjecture of K. Murasugi, Pacific J. Math. 124 (1986), 193-213.

[52] D. Matei, Fundamental groups of links and arrangements: characteristic varieties, resonance varieties, and finite index subgroups, Ph.D. thesis, Northeastern University, Boston, MA, 1999.

[53] D. Matei, A. Suciu, Homotopy types of complements of 2-arrangements in $\mathbb{R}^{4}$, Topology 39 (2000), 61-88.

[54] - Cohomology rings and nilpotent quotients of real and complex arrangements, In: Arrangements-Tokyo 1998 (M. Falk, H. Terao, eds.), Adv. Stud. Pure Math., vol. 27, Math. Soc. Japan, Tokyo, 2000, pp. 185-215.

[55] Hall invariants, homology of subgroups, and characteristic varieties, arXiv: math.GR/0010046.

[56] K. Murasugi, On Milnor's invariants for links. II. The Chen groups, Trans. Amer. Math. Soc. 148 (1970), 41-61.

[57] M. F. Newman, C. Schneider, A. Shalev, The entropy of graded algebras, J. Algebra 223 (2000), 85-100.

[58] S. P. Novikov, Bloch homology, critical points of functions, and closed 1-forms, Dokl. Akad. Nauk. SSSR 287 (1986), 1321-1324; English transl., Soviet Math. Dokl. 33 (1986), $551-555$.

[59] P. Orlik, H. Terao, Arrangements of hyperplanes, Grundlehren Math. Wiss., vol. 300, Springer-Verlag, Berlin, 1992.

[60] - Arrangements and hypergeometric integrals, preprint, 2000.

[61] S. Papadima, S. Yuzvinsky, On rational $K[\pi, 1]$ spaces and Koszul algebras, J. Pure Appl. Alg. 144 (1999), 156-167.

[62] I. Peeva, Hyperplane arrangements and linear strands in resolutions, Trans. Amer. Math. Soc. (to appear). 
[63] R. Randell The fundamental group of the complement of a union of complex hyperplanes, Invent. Math. 69 (1982), 103-108. Correction, Invent. Math. 80 (1985), 467-468.

[64] _ Homotopy and group cohomology of arrangements, Top. Appl. 78 (1997), 201213.

[65] G. Rybnikov, On the fundamental group of the complement of a complex hyperplane arrangement, DIMACS Tech. Report 94-13 (1994), pp. 33-50, arXiv:math.AG/9805056.

[66] M. Sakuma, Homology of abelian coverings of links and spatial graphs, Canad. J. Math. 47 (1995), 201-224.

[67] P. Sarnak, S. Adams, Betti numbers of congruence groups, Israel J. Math. 88 (1994), 31-72. With an appendix by Ze'ev Rudnick.

[68] V. Schechtman, A. Varchenko, Arrangements of hyperplanes and Lie algebra homology, Invent. Math. 106 (1991), 139-194.

[69] B. Shelton, S. Yuzvinsky, Koszul algebras from graphs and hyperplane arrangements, J. London Math. Soc. 56 (1997), 477-490.

[70] C. Simpson, Higgs bundles and local systems, Inst. Hautes Études Sci. Publ. Math. 75 (1992), 5-95.

[71] A. Suciu, Translated tori in the characteristic varieties of complex hyperplane arrangements, Topology Appl. (to appear), arXiv:math.AG/9912227.

[72] I. Tayama, First Betti numbers of abelian coverings of the complex projective plane branched over line configurations, J. Knot Theory Ramifications 9 (2000), 271-284.

[73] E. Westlund, The boundary manifold of an arrangement, Ph.D. thesis, University of Wisconsin, Madison, WI, 1997.

[74] S. Yuzvinsky, Orlik-Solomon algebras in algebra and topology, Russian Math. Surveys (to appear).

[75] K. Zuo, Kummer-Überlagerungen algebraischer Flächen, Bonner Mathematische Schriften, vol. 193, Bonn, 1989.

Department of Mathematics, Northeastern University, Boston, MA 02115

E-mail address: alexsuciu@neu.edu

$U R L:$ http://www.math.neu.edu/ suciu 